Review

\title{
Compilation of Secondary Metabolites from Bidens pilosa $\mathbf{L}$.
}

Fabiana Lima Silva ${ }^{1,2, *}$, Dominique Corinne Hermine Fischer ${ }^{2}$, Josean Fechine Tavares ${ }^{1}$, Marcelo Sobral Silva ${ }^{1}$, Petronio Filgueiras de Athayde-Filho ${ }^{1}$ and Jose Maria Barbosa-Filho ${ }^{1, *}$

1 Laboratório de Tecnologia Farmacêutica, Universidade Federal da Paraíba, Cx. Postal 5009, 58051970, João Pessoa, PB, Brazil; E-Mails: josean@1tf.ufpb.br (J.F.T.); marcelo.ufpb@gmail.com (M.S.S.); athayde-filho@quimica.ufpb.br (P.F.A.F.)

2 Departamento de Farmácia, Faculdade de Ciências Farmacêuticas, Universidade de São Paulo, Av. Prof. Lineu Prestes, Bloco 15, 05580-900, São Paulo, SP, Brazil; E-Mail: domi@usp.br (D.C.H.F.)

* Authors to whom correspondence should be addressed; E-Mails: falimasilva@hotmail.com (F.L.S.); jbarbosa@1tf.ufpb.br (J.M.B.F.); Tel./Fax: + 55-83-3216-7364 (J.M.B.F.).

Received: 11 November 2010; in revised form: 13 January 2011 / Accepted: 24 January 2011 / Published: 26 January 2011

\begin{abstract}
Bidens pilosa L. is a cosmopolitan annual herb, known for its traditional use in treating various diseases and thus much studied for the biological activity of its extracts, fractions and isolated compounds. Polyacetylenes and flavonoids, typical metabolite classes in the Bidens genus, predominate in the phytochemistry of B. pilosa. These classes of compounds have great taxonomic significance. In the Asteraceae family, the acetylene moiety is widely distributed in the Heliantheae tribe and some representatives, such as 1phenylhepta-1,3,5-triyne, are noted for their biological activity and strong long-wave UV radiation absorbance. The flavonoids, specifically aurones and chalcones, have been reported as good sub-tribal level markers. Natural products from several other classes have also been isolated from different parts of B. pilosa. This review summarizes the available information on the 198 natural products isolated to date from B. pilosa.
\end{abstract}

Keywords: Bidens pilosa; Asteraceae; natural products; flavonoids; polyacetylenes 


\section{Introduction}

The genus Bidens (Asteraceae: Heliantheae) comprises about 240 species with cosmopolitan distribution [1]. Many of these species have been investigated chemically to contribute to the classification of Asteraceae [2-4]. Interesting relationships within the Heliantheae, as well as its relationship with other tribes have been proposed on the basis of various types of compounds found in the tribe, especially acetylenes, sesquiterpene lactones and flavonoids [4,5]. The interest in these classes of compounds also has gone beyond chemotaxonomy. The biological activities, including antiparasitic, antifungal and antioxidant properties, of the predominant components in the tribe Heliantheae have been widely reported, and the investigation of these species for the discovery of new active compounds has expanded [6-12].

Bidens pilosa L. (Figure 1) stands out among the species of the genus due to the large number of natural products characterized in it and the biological activities reported for its extracts, fractions and compounds. Therefore, in continuation of our research on bioactive molecules from the various species of the different families cited [13-43], we offer this compilation of the chemical constituents of B. pilosa.

\section{Bidens pilosa L.}

B. pilosa is an annual, erect and ruderal herb originating from South America and now found in almost all tropical and subtropical region countries [44-46]. It grows to a height of up to $1.5 \mathrm{~m}$, branching from the base and its yellow flowers have 5-15 mm diameter [44,46].

Figure 1. Bidens pilosa L.

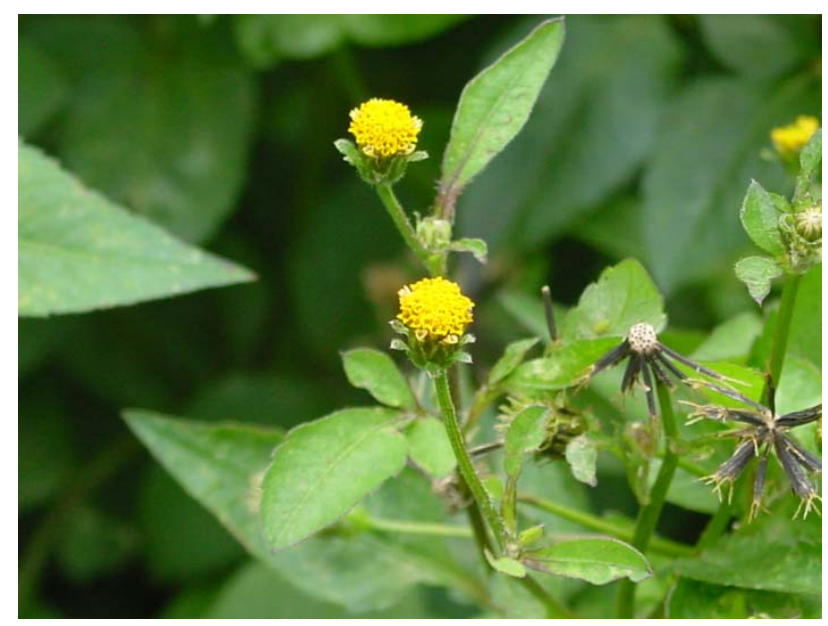

It is a cosmopolitan herb, considered invasive of annual and perennial crops and widely distributed in disturbed areas and along roadsides in tropical and subtropical climates [46]. Nevertheless, this plant is commonly used in the traditional medicine. In Martinique, the decoction of the whole plant is used for its anti-inflammatory and hypoglycemic effects [47]. Aqueous preparations of the leaves are used by Zulu people for the treatment of dysentery, diarrhea and colic [48]. B. pilosa has been popularly used in China as a herbal tea ingredient or in traditional medicine for treating various disorders, such as diabetes, inflammation, enteritis, bacillary dysentery and pharyngitis [49]. In Brazil, it is widely 
used as a folk medicine by indigenous people to treat a variety of illnesses including pain, fever, angina, diabetes, edema, infections and inflammation [50,51]. In addition, in the Amazon and regions in the South of Brazil, hydroalcoholic solutions of $B$. pilosa roots are also regarded as useful in the treatment of malaria [52] and even tumors [53].

Studies of B. pilosa plant extracts have shown it has anti-hyperglycemic [54,55], antihypertensive [56-58], antiulcerogenic [45], hepatoprotective [59], antipyretic [60], immunosuppressive and antiinflammatory [8,61,62], anti-leukemic [63,64], anti-malarial [50], anti-bacterial [48], antioxidant $[65,66]$ and antitumor [67] effects. These proven biological activities have led countries like Brazil to include $B$. pilosa in the official list of medicinal plants with potential for development of herbal use by the public health system [68].

Because the biological activities of some extracts and fractions obtained from different parts of $B$. pilosa, several isolated constituents of the plant have been studied, referring to anti-inflammatory activity, immunosuppressive [44,49,61,69,70], hepatoprotective [59], anti-bacterial [44,71], antifungal [71] anti-malarial [50,71,72], anticancer [72], antiparasitic [73], anti-hyperglycemic activities [49,54,70,74-76], anti-angiogenic [77,78], antioxidant [79] and cercaricidal [80].

\section{The Phytochemistry of Bidens pilosa $\mathrm{L}$.}

B. pilosa has been extensively studied since the early 1900s. Among the classes of compounds reported polyacetylenes and flavonoids, typical metabolite classes in the Bidens genus, predominate $[4,81]$. These are also the most reported classes of compounds when referring to the biological activities $[49,50,54,61,74,75,82,83]$. A number of earlier studies also have reported the isolation of sterols $[44,84,85]$, terpenoids [46,85,86], phenylpropanoids [62,83,87-90] and hydrocarbons $[44,85,91]$.

There have been a few reviews of $B$. pilosa $[6,51,92,93]$, however the phytochemical data have not included all classes of metabolites. To date almost 198 compounds have been described from this species. These secondary metabolites are listed in Table 1, where they were grouped based on the classification adopted by a standard reference work, the Dictionary of Natural Products [94].

The order begins with the structurally most simple metabolites, derived from aliphatic natural produts (branched, unbranched, saturated or unsaturated hydrocarbons), and among these, the acetylenes are highlighted. Next the derivatives of simple aromatic hydrocarbons and the phenylpropanoids, in which a C3 substituent is attached to the aromatic unit (C6), form a biosynthetically distinct group of aromatic metabolites. The flavonoids, also considered a large group of metabolites in B. pilosa are subdivided into aurones, chalcones, flavanones, flavones and flavonols. The terpenoids group is divided according to the number of carbons, starting in sesquiterpenes and continuing with diterpenes, sterols, triterpenes and finally tetraterpenes. Finally, porphyrins, nitrogen and sulphur-containing natural products, one disaccharide and miscellaneous compounds are arranged. 
Table 1. Compounds isolated from Bidens pilosa $\mathrm{L}$.

\begin{tabular}{|c|c|c|c|c|c|c|}
\hline $\mathbf{N}^{\circ}$. & Name & $\begin{array}{c}\text { Alternative } \\
\text { name }\end{array}$ & Structure & $\begin{array}{c}\text { Plant } \\
\text { part }\end{array}$ & Country & Ref. \\
\hline \multicolumn{7}{|c|}{ Aliphatic natural products } \\
\hline \multicolumn{7}{|c|}{ Saturated unbranched hydrocarbons } \\
\hline 1 & heneicosane & & $\mathrm{CH}_{3}\left(\mathrm{CH}_{2}\right)_{19} \mathrm{CH}_{3}$ & $\mathrm{AP}$ & Tanzania & {$[44]$} \\
\hline 2 & dodosane & & $\mathrm{CH}_{3}\left(\mathrm{CH}_{2}\right)_{20} \mathrm{CH}_{3}$ & $\mathrm{AP}$ & Tanzania & {$[44]$} \\
\hline 3 & tricosane & & $\mathrm{CH}_{3}\left(\mathrm{CH}_{2}\right)_{21} \mathrm{CH}_{3}$ & $\mathrm{AP}$ & Tanzania & {$[44]$} \\
\hline 4 & tetracosane & & $\mathrm{CH}_{3}\left(\mathrm{CH}_{2}\right)_{22} \mathrm{CH}_{3}$ & $\mathrm{AP}$ & Tanzania & [44] \\
\hline 5 & pentacosane & & $\mathrm{CH}_{3}\left(\mathrm{CH}_{2}\right)_{23} \mathrm{CH}_{3}$ & $\mathrm{AP}$ & Tanzania & {$[44]$} \\
\hline 6 & hexacosane & & $\mathrm{CH}_{3}\left(\mathrm{CH}_{2}\right)_{24} \mathrm{CH}_{3}$ & $\mathrm{AP}$ & Tanzania & {$[44]$} \\
\hline 7 & heptacosane & & $\mathrm{CH}_{3}\left(\mathrm{CH}_{2}\right)_{25} \mathrm{CH}_{3}$ & $\mathrm{AP}$ & Tanzania & [44] \\
\hline \multirow{2}{*}{8} & \multirow{2}{*}{ octacosane } & & \multirow{2}{*}{$\mathrm{CH}_{3}\left(\mathrm{CH}_{2}\right)_{26} \mathrm{CH}_{3}$} & $\mathrm{NF}$ & Taiwan & {$[91]$} \\
\hline & & & & $\mathrm{AP}$ & Tanzania & {$[44]$} \\
\hline \multirow{2}{*}{9} & \multirow{2}{*}{ nonocosane } & & \multirow{2}{*}{$\mathrm{CH}_{3}\left(\mathrm{CH}_{2}\right)_{27} \mathrm{CH}_{3}$} & $\mathrm{NF}$ & Taiwan & {$[91]$} \\
\hline & & & & $\mathrm{AP}$ & Tanzania & {$[44]$} \\
\hline \multirow{2}{*}{10} & \multirow{2}{*}{ triacontane } & & \multirow{2}{*}{$\mathrm{CH}_{3}\left(\mathrm{CH}_{2}\right)_{28} \mathrm{CH}_{3}$} & $\mathrm{NF}$ & Taiwan & {$[91]$} \\
\hline & & & & $\mathrm{AP}$ & Tanzania & {$[44]$} \\
\hline \multirow{2}{*}{11} & \multirow{2}{*}{ hentriacontane } & & \multirow{2}{*}{$\mathrm{CH}_{3}\left(\mathrm{CH}_{2}\right)_{29} \mathrm{CH}_{3}$} & $\mathrm{NF}$ & Taiwan & {$[91]$} \\
\hline & & & & $\mathrm{AP}$ & Tanzania & {$[44]$} \\
\hline \multirow{2}{*}{12} & \multirow{2}{*}{ dotriacontane } & & \multirow{2}{*}{$\mathrm{CH}_{3}\left(\mathrm{CH}_{2}\right)_{30} \mathrm{CH}_{3}$} & $\mathrm{NF}$ & Taiwan & {$[91]$} \\
\hline & & & & $\mathrm{AP}$ & Tanzania & {$[44]$} \\
\hline \multirow{2}{*}{13} & \multirow{2}{*}{ tritriacontane } & & \multirow{2}{*}{$\mathrm{CH}_{3}\left(\mathrm{CH}_{2}\right)_{31} \mathrm{CH}_{3}$} & NF & Taiwan & {$[91]$} \\
\hline & & & & $\mathrm{AP}$ & Tanzania & {$[44]$} \\
\hline \multicolumn{7}{|c|}{ Saturated unbranched alcohols } \\
\hline 14 & 2-butoxy-ethanol & & $\mathrm{CH}_{3}\left(\mathrm{CH}_{2}\right)_{3} \mathrm{OCH}_{2} \mathrm{CH}_{2} \mathrm{OH}$ & EP & Taiwan & {$[85]$} \\
\hline 15 & tetracosan-1-ol & & $\mathrm{CH}_{3}\left(\mathrm{CH}_{2}\right)_{22} \mathrm{CH}_{2} \mathrm{OH}$ & AP & Tanzania & [44] \\
\hline 16 & hexacosan-1-ol & & $\mathrm{CH}_{3}\left(\mathrm{CH}_{2}\right)_{24} \mathrm{CH}_{2} \mathrm{OH}$ & AP & Tanzania & [44] \\
\hline 17 & 1-octacosanol & & $\mathrm{CH}_{3}\left(\mathrm{CH}_{2}\right)_{26} \mathrm{CH}_{2} \mathrm{OH}$ & $\mathrm{AP}$ & Tanzania & {$[44]$} \\
\hline 18 & 1-hentriacontanol & & $\mathrm{CH}_{3}\left(\mathrm{CH}_{2}\right)_{29} \mathrm{CH}_{2} \mathrm{OH}$ & NF & Taiwan & [91] \\
\hline \multicolumn{7}{|c|}{ Saturated unbranched carboxylic acids } \\
\hline 19 & tetradecanoic acid & myristic acid & $\mathrm{CH}_{3}\left(\mathrm{CH}_{2}\right)_{12} \mathrm{CO}_{2} \mathrm{H}$ & AP & Tanzania & [44] \\
\hline 20 & hexadecanoic acid & palmitic acid & $\mathrm{CH}_{3}\left(\mathrm{CH}_{2}\right)_{14} \mathrm{CO}_{2} \mathrm{H}$ & $\mathrm{AP}$ & Tanzania & [44] \\
\hline 21 & octadecanoic acid & stearic acid & $\mathrm{CH}_{3}\left(\mathrm{CH}_{2}\right)_{16} \mathrm{CO}_{2} \mathrm{H}$ & AP & Tanzania & [44] \\
\hline 22 & eicosanoic acid & arachidic acid & $\mathrm{CH}_{3}(\mathrm{CH} 2)_{18} \mathrm{CO}_{2} \mathrm{H}$ & AP & Tanzania & {$[44]$} \\
\hline 23 & docosanoid acid & behenic acid & $\mathrm{CH}_{3}(\mathrm{CH} 2)_{20} \mathrm{CO}_{2} \mathrm{H}$ & LF & not stated & [84] \\
\hline \multicolumn{7}{|c|}{ Unbranched aliphatic carboxylic acid esters } \\
\hline 24 & 2-butenedioic acid & & & $\begin{array}{l}\text { AP } \\
\text { AP }\end{array}$ & $\begin{array}{l}\text { China } \\
\text { China }\end{array}$ & $\begin{array}{l}{[121]} \\
{[102]}\end{array}$ \\
\hline 25 & $\begin{array}{l}\text { (Z)-9-octadecenoic } \\
\text { acid }\end{array}$ & oleic acid & & AP & Tanzania & [44] \\
\hline 26 & $\begin{array}{l}(E) \text {-9-octadecenoic } \\
\text { acid }\end{array}$ & elaidic acid & & LF & not stated & [84] \\
\hline 27 & $\begin{array}{l}(Z, Z)-9,12- \\
\text { octadecadienoic acid }\end{array}$ & $\begin{array}{l}\text { linolic } \\
\text { acid/linoleic } \\
\text { acid }\end{array}$ & & $\begin{array}{l}\mathrm{AP} \\
\mathrm{EP}\end{array}$ & $\begin{array}{l}\text { Tanzania } \\
\text { Taiwan }\end{array}$ & $\begin{array}{l}{[44]} \\
{[85]} \\
\end{array}$ \\
\hline
\end{tabular}


Table 1. Cont.

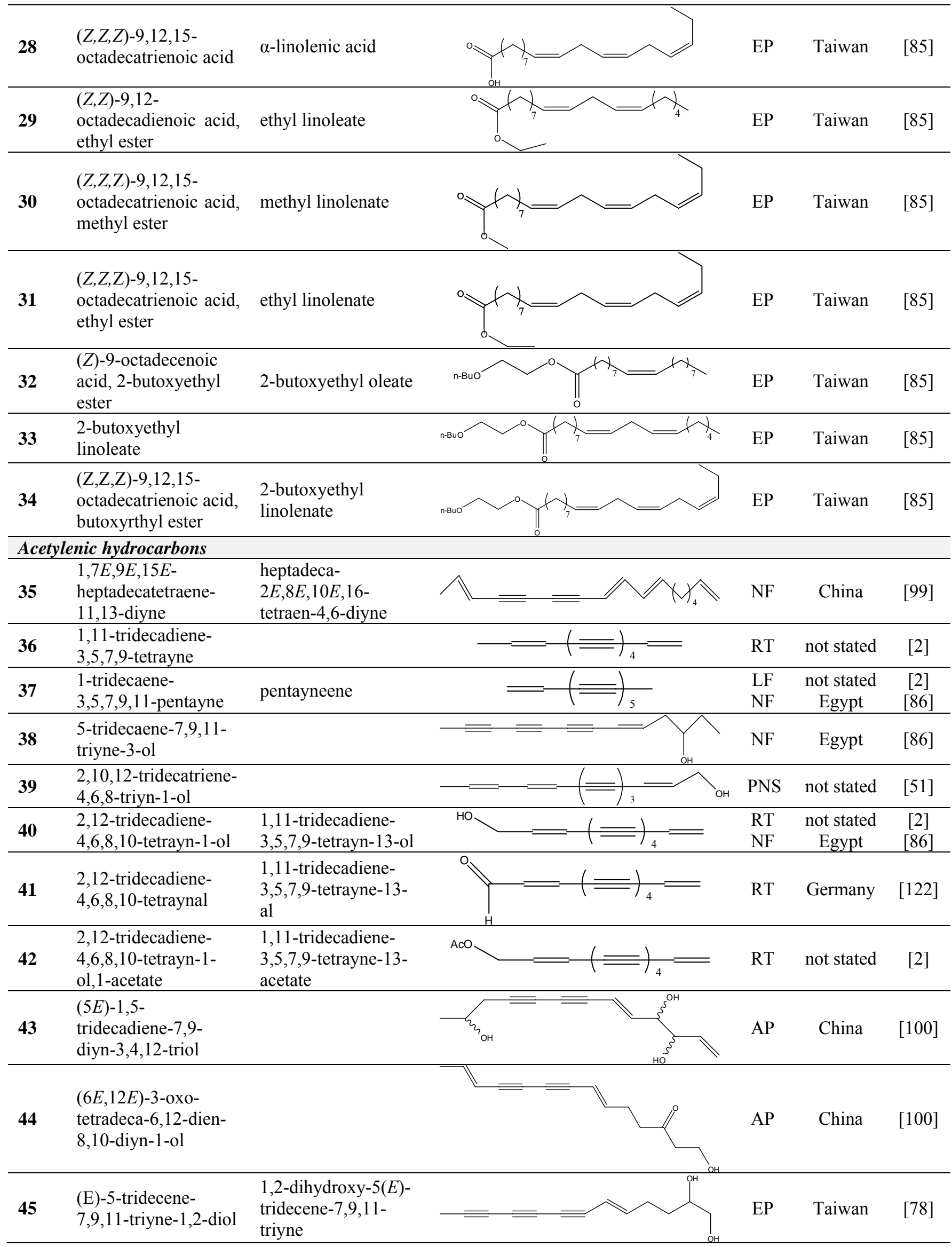


Table 1. Cont.

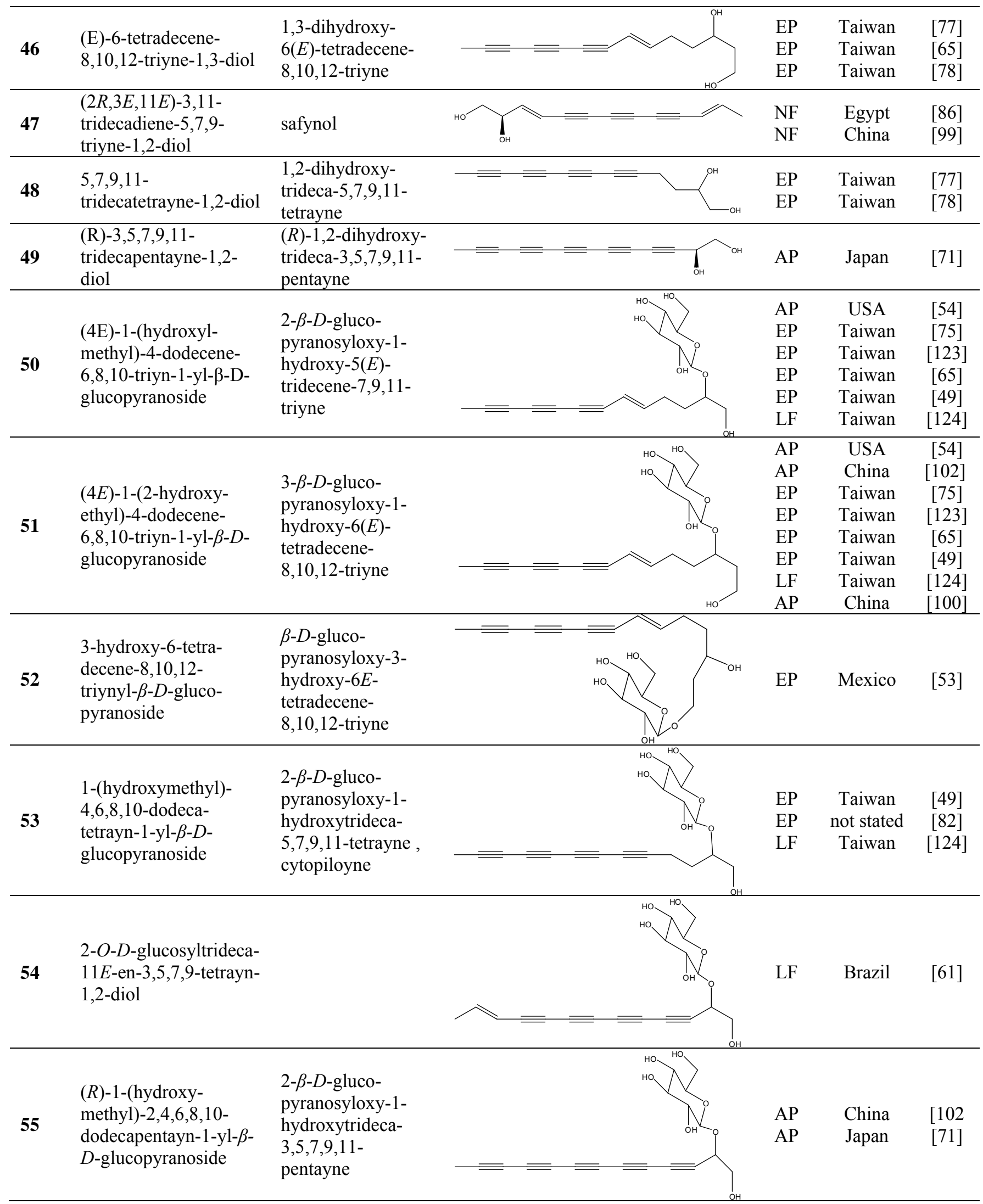


Table 1. Cont.

1-[[(carboxyacetyl)oxy]methyl]-

4,6,8,10-dodecatetraynyl- $\beta-D$ -

glucopyranoside

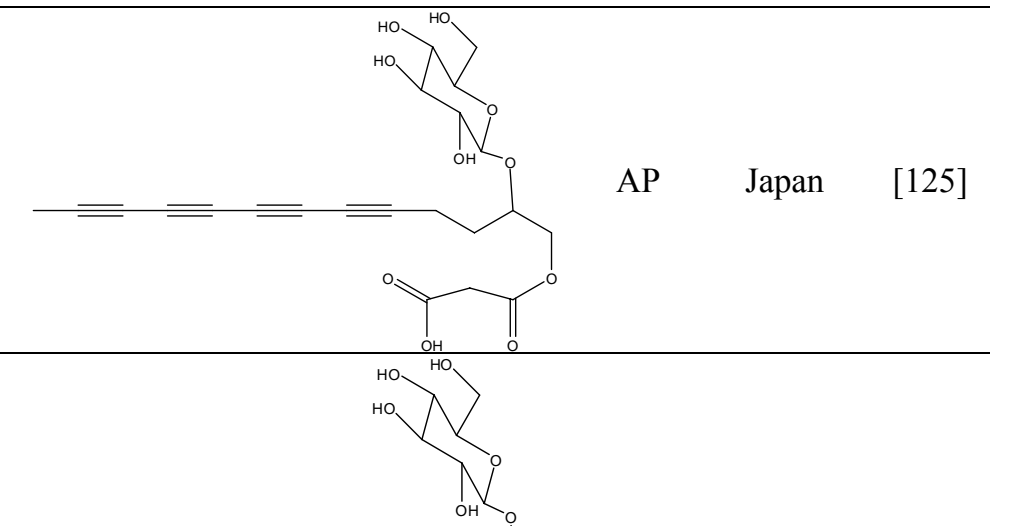

(4E)-1-[[(carboxyacetyl)oxy]-methyl]-4dodecene-6,8,10triynyl- $\beta$ - $D$-glucopyranoside

(4E)-1-[[(carboxyacetyl)oxy]-ethyl]-4dodecene-6,8,10triynyl- $\beta$ - $D$-glucopyranoside

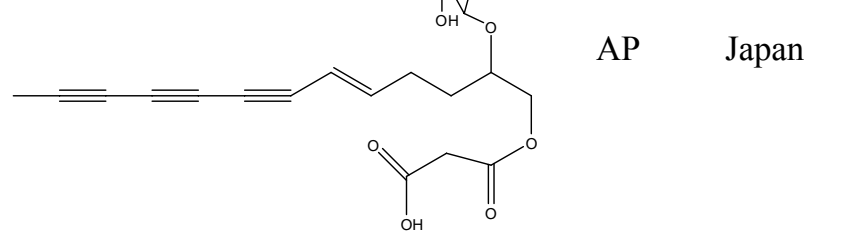

\begin{tabular}{|c|c|c|c|c|c|}
\hline 59 & $\begin{array}{l}\text { (5E)-5-heptene-1,3- } \\
\text { diyn-1-yl-benzene }\end{array}$ & $\begin{array}{l}\text { 1-phenylhepta-1,3- } \\
\text { diyn-5-en }\end{array}$ & EP & Taiwan & {$[85]$} \\
\hline 60 & $\begin{array}{l}\text { 7-phenyl-2 }(E) \text { - } \\
\text { heptene-4,6-diyn-1-ol }\end{array}$ & & $\begin{array}{l}\text { RT } \\
\text { AP }\end{array}$ & $\begin{array}{l}\text { not stated } \\
\text { China }\end{array}$ & $\begin{array}{c}{[2]} \\
{[100]}\end{array}$ \\
\hline 61 & $\begin{array}{l}\text { 7-phenyl-2(E)- } \\
\text { heptene-4,6-diyn-1-ol- } \\
\text { acetate }\end{array}$ & & $\begin{array}{l}\text { RT } \\
\text { RT } \\
\text { RT } \\
\end{array}$ & $\begin{array}{c}\text { not stated } \\
\text { Brazil } \\
\text { Brazil } \\
\end{array}$ & $\begin{array}{c}{[2]} \\
{[50]} \\
{[52]}\end{array}$ \\
\hline 62 & $\begin{array}{l}\text { 7-phenyl-4,6- } \\
\text { heptadiyn-2-ol }\end{array}$ & (-)-pilosol A & $\begin{array}{l}\mathrm{EP} \\
\mathrm{AP}\end{array}$ & $\begin{array}{l}\text { Taiwan } \\
\text { China }\end{array}$ & $\begin{array}{c}{[85]} \\
{[100]}\end{array}$ \\
\hline 63 & $\begin{array}{l}\text { 7-phenylhepta-4,6- } \\
\text { diyn-1,2-diol }\end{array}$ & & AP & China & {$[100]$} \\
\hline 64 & $\begin{array}{l}\text { 1,3,5-heptatriyn-1-yl- } \\
\text { benzene }\end{array}$ & $\begin{array}{l}\text { 1-phenylhepta- } \\
\text { 1,3,5-triyne }\end{array}$ & $\begin{array}{c}\text { LF } \\
\text { LTC } \\
\text { AP } \\
\text { AP } \\
\text { EP } \\
\text { RT } \\
\text { AP } \\
\end{array}$ & $\begin{array}{c}\text { not stated } \\
\text { not stated } \\
\text { Tanzania } \\
\text { China } \\
\text { Taiwan } \\
\text { Brazil } \\
\text { China } \\
\end{array}$ & $\begin{array}{c}2] \\
{[97]} \\
{[44]} \\
{[121]} \\
{[85]} \\
{[52]} \\
{[100]} \\
\end{array}$ \\
\hline 65 & $\begin{array}{l}\text { 7-phenyl-2,4,6- } \\
\text { heptatriyn-1-ol }\end{array}$ & & $\begin{array}{l}\mathrm{LF} \\
\mathrm{AP}\end{array}$ & $\begin{array}{l}\text { not stated } \\
\text { China }\end{array}$ & $\begin{array}{c}{[2]} \\
{[100]}\end{array}$ \\
\hline 66 & $\begin{array}{l}\text { 7-phenyl-2,4,6- } \\
\text { heptatriyn-1-ol-acetate }\end{array}$ & & LF & not stated & {$[2]$} \\
\hline 67 & $\begin{array}{l}\text { 5-(2-phenylethynyl)-2- } \\
\text { thiophene methanol }\end{array}$ & & $\mathrm{AP}$ & China & {$[100]$} \\
\hline
\end{tabular}


Table 1. Cont.

\begin{tabular}{|c|c|c|c|c|c|}
\hline 68 & $\begin{array}{l}\text { 5-(2-phenylethynyl)- } \\
2 \beta \text {-glucosylmethyl- } \\
\text { thiophene }\end{array}$ & & AP & China & {$[100]$} \\
\hline \multicolumn{6}{|c|}{ Simple aromatic hydrocarbons } \\
\hline \multicolumn{6}{|c|}{ Simple phenols } \\
\hline 69 & 1,2-benzenediol & pyrocatechin & EP & Japan & {$[87]$} \\
\hline 70 & $\begin{array}{l}\text { 4-ethyl-1,2- } \\
\text { benzenediol }\end{array}$ & pyrocatechol & EP & Japan & [87] \\
\hline 71 & dimethoxyphenol & & RT & Japan & {$[87]$} \\
\hline 72 & $\begin{array}{l}\text { 4-ethenyl-2-methoxy- } \\
\text { phenol }\end{array}$ & $p$-vinylguaiacol & EP & Japan & {$[87]$} \\
\hline 73 & $\begin{array}{l}\text { 2-hydroxy-6- } \\
\text { methylbenzaldehyde }\end{array}$ & $\begin{array}{l}\text { 6-methyl- } \\
\text { salicylaldehyde }\end{array}$ & EP & Japan & {$[87]$} \\
\hline 74 & benzene-ethanol & 2-phenyl-ethanol & EP & Taiwan & {$[85]$} \\
\hline \multicolumn{6}{|c|}{ Simple aryl aldehydes } \\
\hline 75 & $\begin{array}{l}\text { 4-hydroxy-3-methoxy- } \\
\text { benzaldehyde }\end{array}$ & vanillin & AP & Japan & [87] \\
\hline 76 & $\begin{array}{l}\text { 3-hydroxy-4-methoxy- } \\
\text { benzaldehyde }\end{array}$ & vanillin, iso & LF & Japan & [87] \\
\hline \multicolumn{6}{|c|}{ Simple benzoic acids and their homologues } \\
\hline 77 & $\begin{array}{l}\text { 4-hydroxy-benzoic } \\
\text { acid }\end{array}$ & $\begin{array}{l}p \text {-hydroxybenzoic } \\
\text { acid }\end{array}$ & EP & Japan & [87] \\
\hline 78 & $\begin{array}{l}\text { 2-hydroxy-benzoic } \\
\text { acid }\end{array}$ & salicylic acid & $\begin{array}{c}\mathrm{ST} / \mathrm{R} \\
\mathrm{T}\end{array}$ & Japan & [87] \\
\hline 79 & $\begin{array}{l}\text { 3,4-dihydroxy-benzoic } \\
\text { acid }\end{array}$ & protocatechuic acid & EP & Japan & {$[87]$} \\
\hline 80 & $\begin{array}{l}\text { 4-hydroxy-3-methoxy- } \\
\text { benzoic acid }\end{array}$ & vanillic acid & $\begin{array}{l}\text { AP } \\
\text { RT }\end{array}$ & $\begin{array}{l}\text { Uganda } \\
\text { Japan }\end{array}$ & $\begin{array}{c}{[110]} \\
{[87]}\end{array}$ \\
\hline 81 & $\begin{array}{l}\text { 3,4,5-trihydroxy- } \\
\text { benzoic acid }\end{array}$ & gallic acid & EP & China & [126] \\
\hline
\end{tabular}


Table 1. Cont.

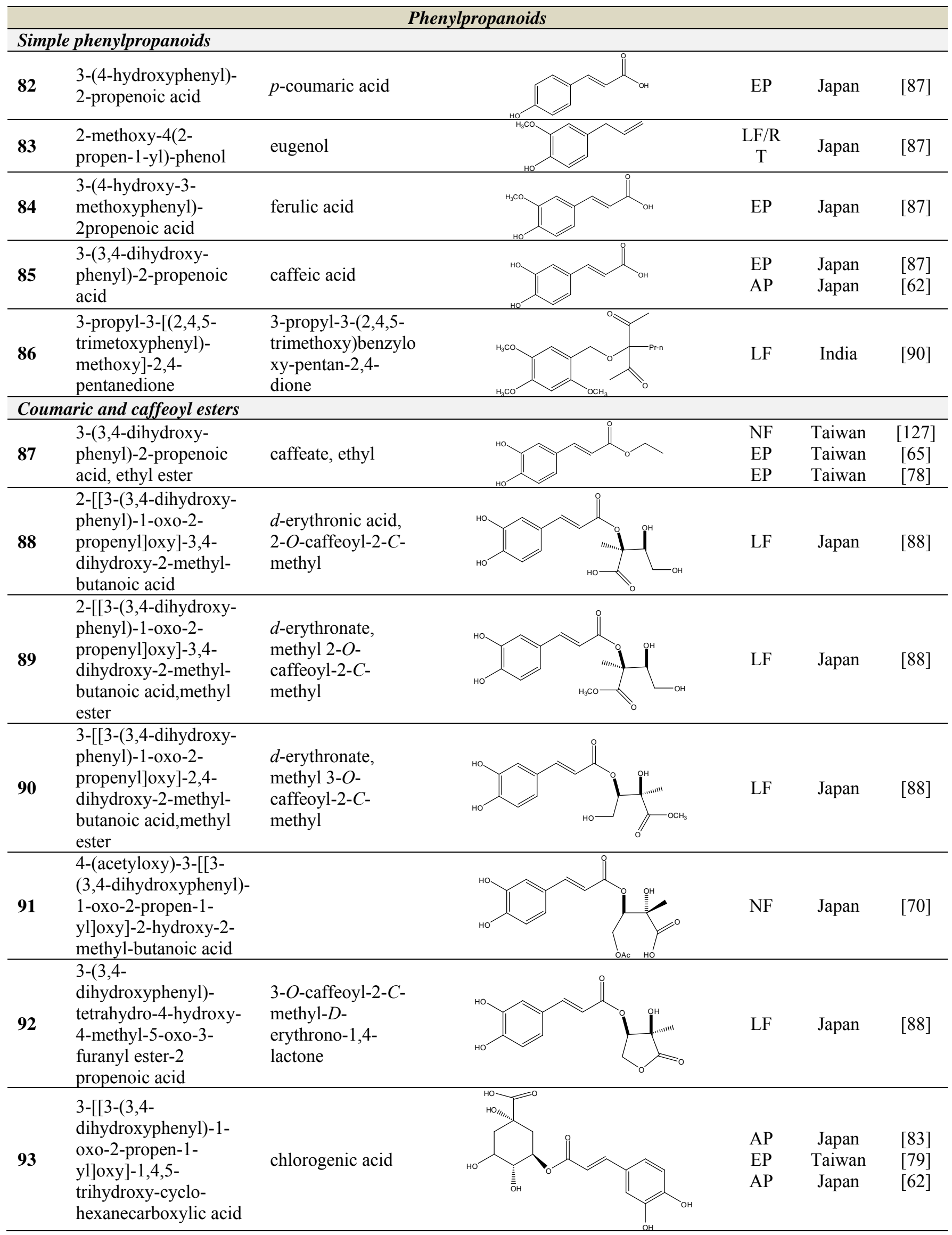


Table 1. Cont.

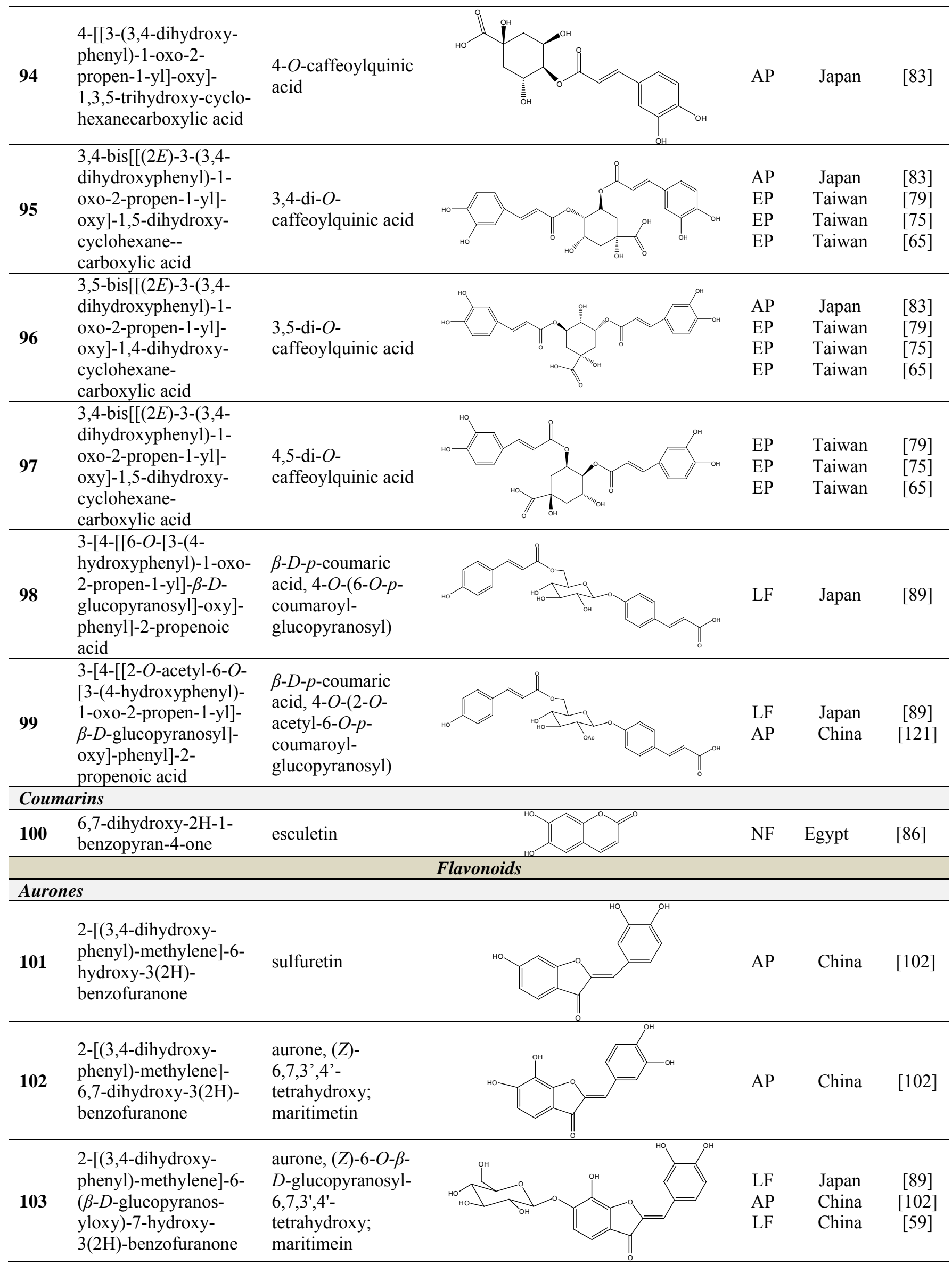


Table 1. Cont.

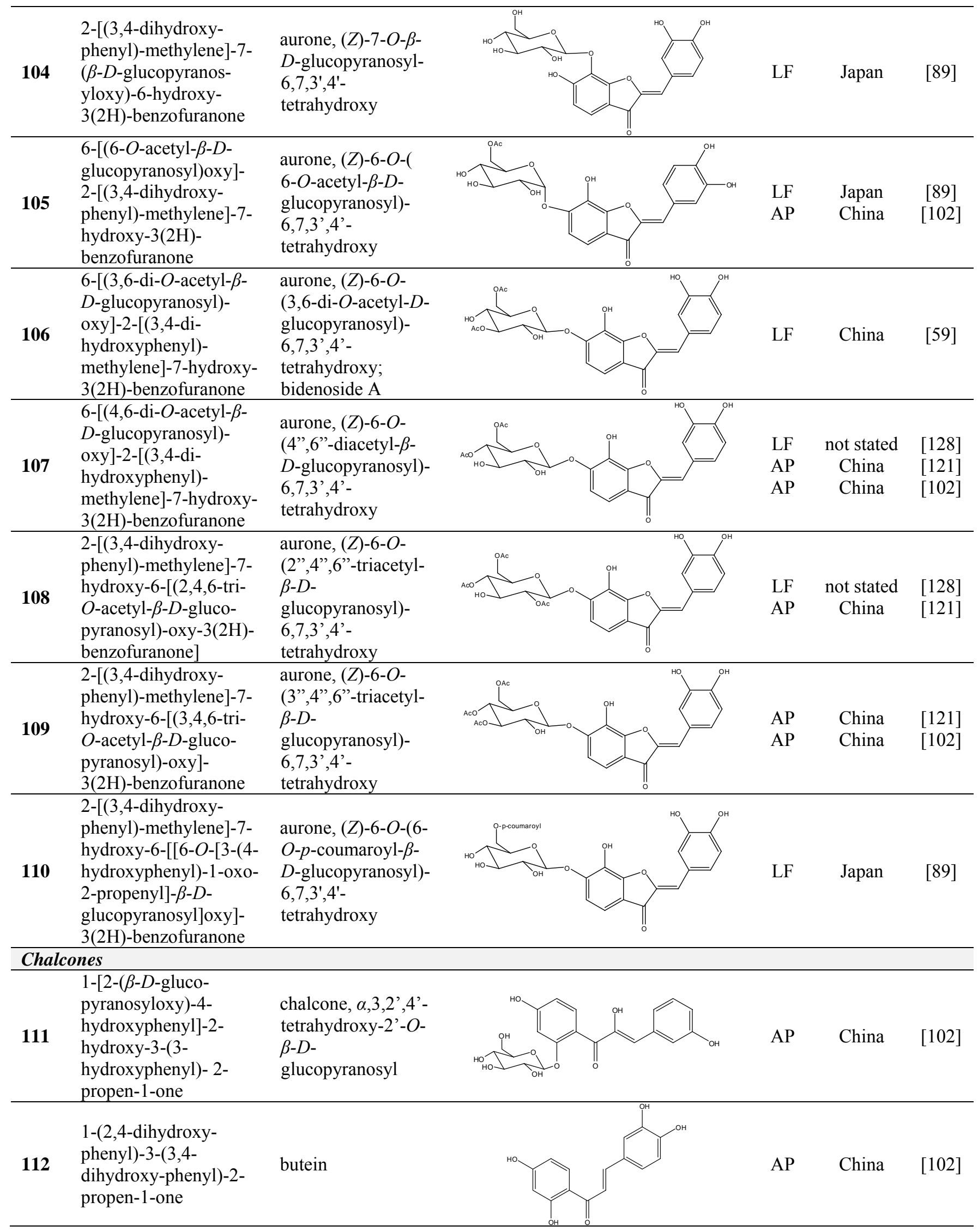


Table 1. Cont.

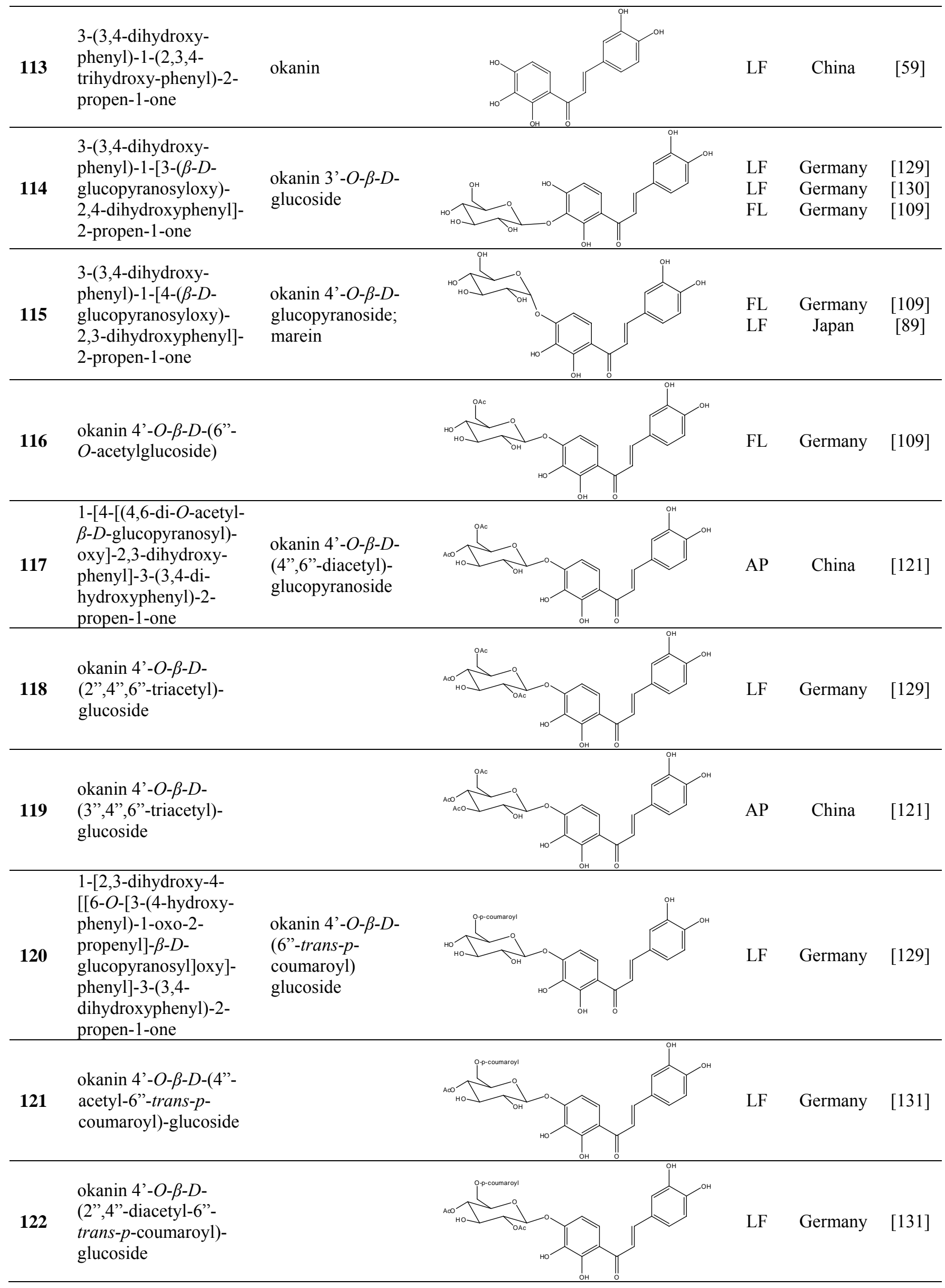


Table 1. Cont.

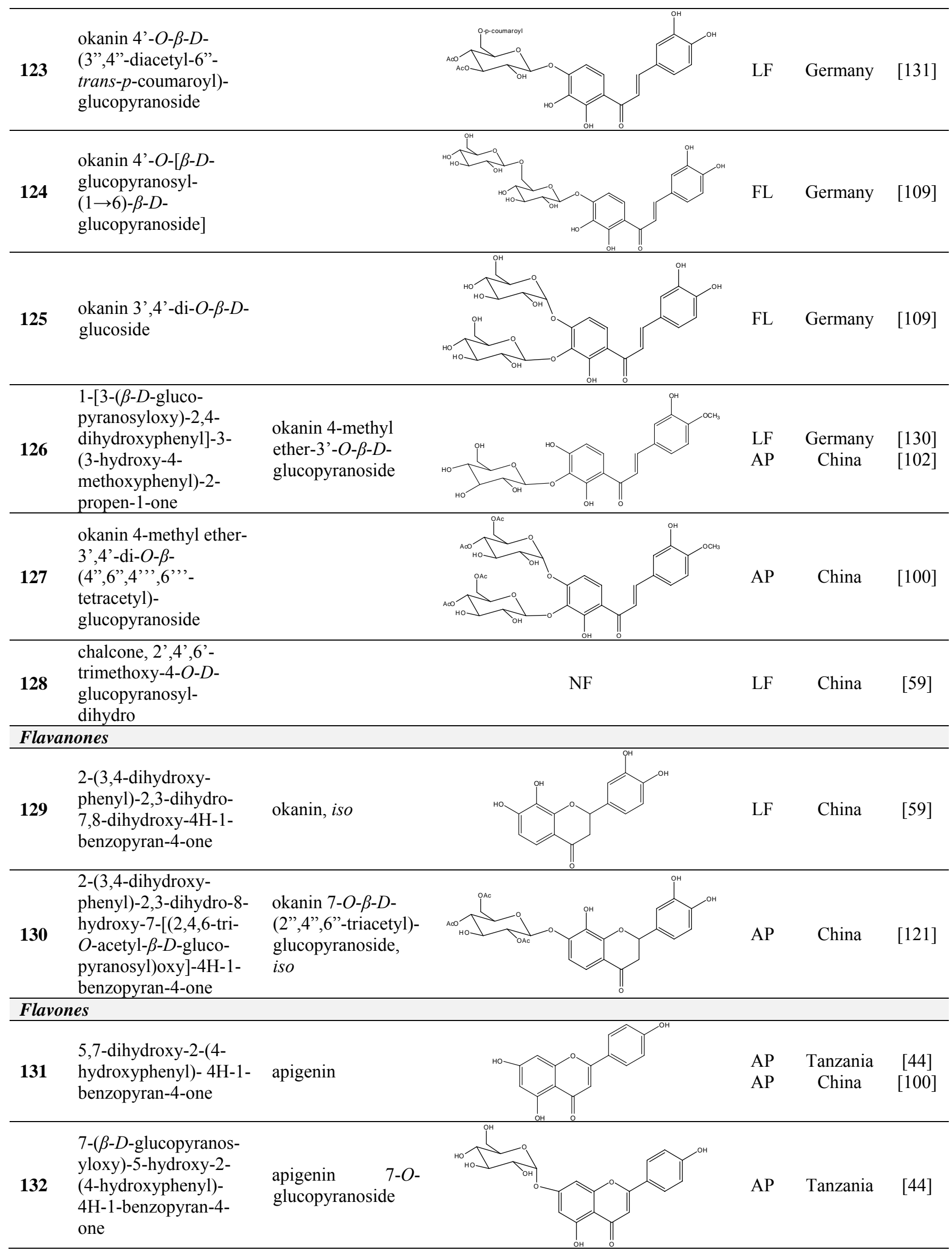


Table 1. Cont.

\begin{tabular}{|c|c|c|c|c|c|}
\hline 133 & $\begin{array}{l}\text { 2-(3,4-dihydroxy- } \\
\text { phenyl)-5,7-dihydroxy- } \\
\text { 4H-1-benzopyran-4- } \\
\text { one }\end{array}$ & luteolin & $\begin{array}{l}\text { AP } \\
\text { AP } \\
\text { AP } \\
\text { AP } \\
\text { AP }\end{array}$ & $\begin{array}{l}\text { Tanzania } \\
\text { China } \\
\text { China } \\
\text { China } \\
\text { Vietnam }\end{array}$ & $\begin{array}{l}{[44]} \\
{[121]} \\
{[102]} \\
{[100]} \\
{[132]}\end{array}$ \\
\hline 134 & $\begin{array}{l}\text { 2-(3,4-dihydroxy- } \\
\text { phenyl)-7- }(\beta-D \text {-gluco- } \\
\text { pyranosyloxy)-5- } \\
\text { hydroxy-4H-1- } \\
\text { benzopyran-4-one }\end{array}$ & $\begin{array}{l}\text { luteolin } 7-O-\beta-D- \\
\text { glucopyranoside }\end{array}$ & $\mathrm{AP}$ & Tanzania & {$[44]$} \\
\hline 135 & $\begin{array}{l}\text { 5,7-dimethoxy-6-(5- } \\
\text { methoxy-6-methyl-4- } \\
\text { oxo-4H-pyran-3-yl)-2- } \\
\text { phenyl-4H-1- } \\
\text { benzopyran-4-one }\end{array}$ & $\begin{array}{l}5-O- \\
\text { methylhoslundin }\end{array}$ & $\mathrm{AP}$ & Uganda & {$[110]$} \\
\hline \multicolumn{6}{|c|}{ Flavonols } \\
\hline 136 & $\begin{array}{l}\text { 3-( } \beta \text { - } D \text {-gluco- } \\
\text { pyranosyloxy })-5,7- \\
\text { dihydroxy-2-(4- } \\
\text { hydroxyphenyl)- } 4 \mathrm{H}-1- \\
\text { benzopyran-4-one }\end{array}$ & $\begin{array}{l}\text { astragalin; } \\
\text { kaempferol-3-O- } \beta \text { - } \\
D \text {-glucopyranoside }\end{array}$ & $\mathrm{AP}$ & China & [102] \\
\hline 137 & $\begin{array}{l}\text { kaempferol 3-(2,3-di- } \\
E \text { - } p \text {-coumaroyl- } \alpha-L- \\
\text { rhamnopyranoside) }\end{array}$ & & $\mathrm{AP}$ & Vietnam & [132] \\
\hline 138 & $\begin{array}{l}\text { 2-(3,4-dihydroxy- } \\
\text { phenyl)-7-( } \beta-D- \\
\text { glucopyranosyloxy)-5- } \\
\text { hydroxy-3,6- } \\
\text { dimethoxy-4H-1- } \\
\text { benzopyran-4-one }\end{array}$ & axillaroside & $\mathrm{AP}$ & China & [100] \\
\hline 139 & $\begin{array}{l}\text { 5,7-dihydroxy-2-(3- } \\
\text { hydroxy-4-methoxy- } \\
\text { phenyl)-3,6-di- } \\
\text { methoxy-4H-1- } \\
\text { benzopyran-4-one }\end{array}$ & centaureidin & $\mathrm{EP}$ & Taiwan & {$[74]$} \\
\hline 140 & $\begin{array}{l}\text { 7-( } \beta \text { - } D \text {-glucopyranos- } \\
\text { yloxy)-5-hydroxy-2- } \\
\text { (3-hydroxy-4- } \\
\text { methoxyphenyl)-3,6- } \\
\text { dimethoxy-4H-1- } \\
\text { benzopyran-4-one }\end{array}$ & centaurein & $\begin{array}{l}\mathrm{AP} \\
\mathrm{EP} \\
\mathrm{EP}\end{array}$ & $\begin{array}{c}\text { Japan } \\
\text { Taiwan } \\
\text { Taiwan }\end{array}$ & $\begin{array}{l}{[83]} \\
{[79]} \\
{[74]}\end{array}$ \\
\hline 141 & & eupatorin, iso & $\mathrm{NF}$ & China & [99] \\
\hline 142 & $\begin{array}{l}\text { 2-(3,4-dimethoxy- } \\
\text { phenyl)-7- }(\beta-D- \\
\text { glucopyranosyloxy)- } \\
3,5 \text {-dihydroxy-8- } \\
\text { methoxy-4H-1- } \\
\text { benzopyran-4-one }\end{array}$ & & $\mathrm{NF}$ & Japan & [70] \\
\hline
\end{tabular}


Table 1. Cont.

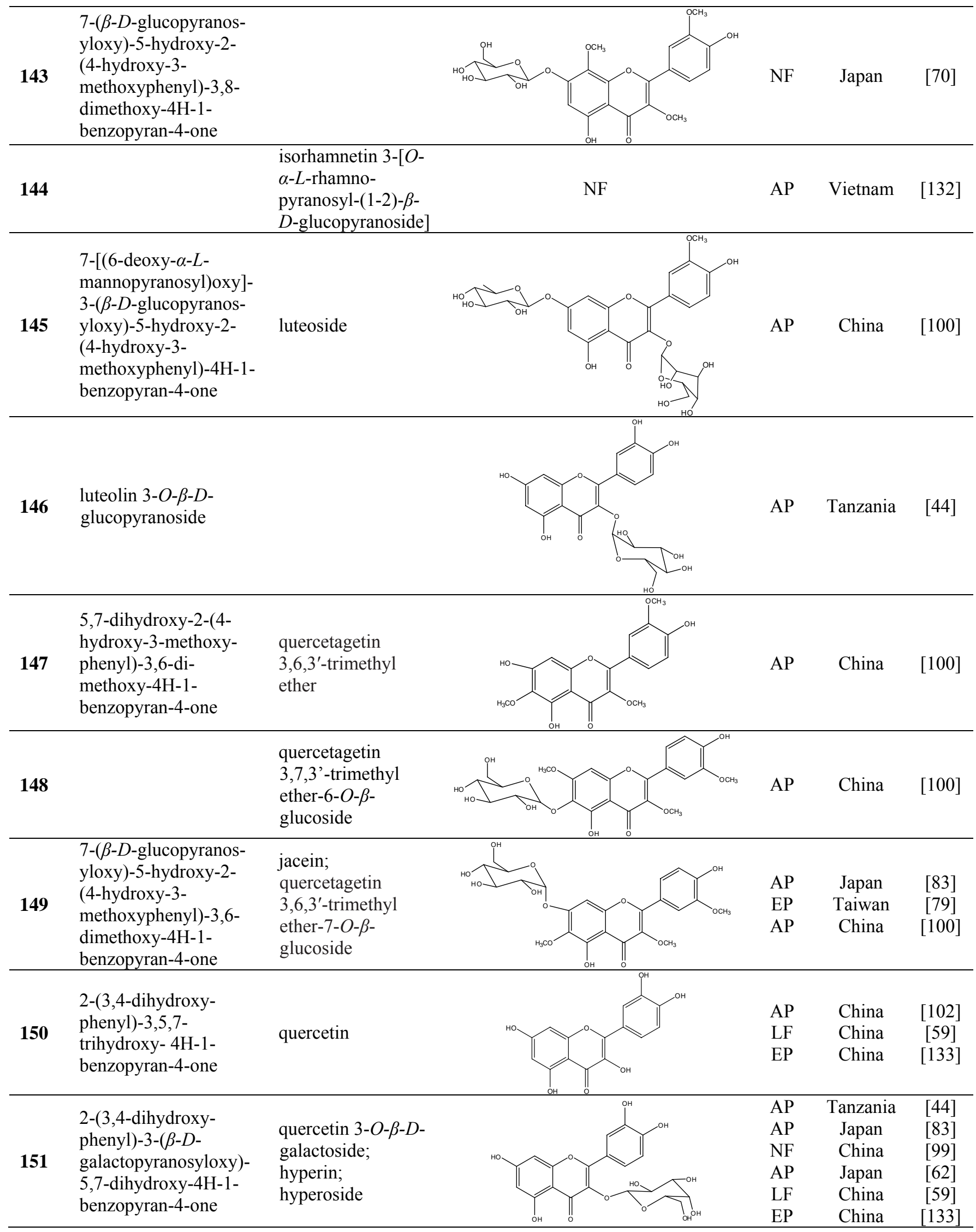


Table 1. Cont.

\begin{tabular}{|c|c|c|c|c|c|}
\hline 152 & $\begin{array}{l}\text { 2-(3,4-dihydroxy- } \\
\text { phenyl)-3-( } \beta \text { - } D- \\
\text { glucopyranosyloxy)- } \\
5,7 \text {-dihydroxy-4H-1- } \\
\text { benzopyran-4-one }\end{array}$ & $\begin{array}{l}\text { quercetin } 3-O-\beta-D- \\
\text { glucopyranoside }\end{array}$ & $\begin{array}{l}\text { AP } \\
\text { LF } \\
\text { AP } \\
\text { AP }\end{array}$ & $\begin{array}{c}\text { Tanzania } \\
\text { Japan } \\
\text { China } \\
\text { Japan }\end{array}$ & $\begin{array}{r}{[44]} \\
{[89]} \\
{[102]} \\
{[62]}\end{array}$ \\
\hline 153 & $\begin{array}{l}\text { 2-(3,4-dihydroxy- } \\
\text { phenyl)-5,7-dihydroxy- } \\
\text { 4-oxo-4H-1-benzo- } \\
\text { pyran-3-yl- } \beta \text { - } D \text { - } \\
\text { glucopyranosiduronic } \\
\text { acid }\end{array}$ & $\begin{array}{l}\text { quercetin } 3-O-\beta-D- \\
\text { glucuronopyranosi } \\
\text { de }\end{array}$ & $\begin{array}{l}\text { AP } \\
\text { AP }\end{array}$ & $\begin{array}{c}\text { Tanzania } \\
\text { Japan }\end{array}$ & $\begin{array}{l}{[44]} \\
{[83]}\end{array}$ \\
\hline 154 & $\begin{array}{l}\text { 3-[[6-O-(6-deoxy- } \alpha \text {-L- } \\
\text { mannopyranosyl)- } \beta \text {-D- } \\
\text { galactopyranosyl] } 0 x y]- \\
2 \text {-( }(3,4-d i h y d r o x y- \\
\text { phenyl)-5,7-dihydroxy- } \\
4 \text { H-1-benzopyran-4- } \\
\text { one }\end{array}$ & $\begin{array}{l}\text { quercetin 3-O- } \\
\text { robinobioside }\end{array}$ & $\begin{array}{l}\text { AP } \\
\text { EP }\end{array}$ & $\begin{array}{l}\text { Japan } \\
\text { Taiwan }\end{array}$ & $\begin{array}{l}{[83]} \\
{[79]}\end{array}$ \\
\hline 156 & $\begin{array}{l}\text { 7-( } \beta \text { - } D \text {-glucopyranos- } \\
\text { yloxy)-5-hydroxy-2- } \\
\text { (4-hydroxy-3- } \\
\text { methoxyphenyl)-3- } \\
\text { methoxy-4H-1- } \\
\text { benzopyran-4-one }\end{array}$ & $\begin{array}{l}\text { quercetin } 3,3 \text { '- } \\
\text { dimethyl ether } 7-O \text { - } \\
\beta \text { - } D \text { - } \\
\text { glucopyranoside }\end{array}$ & $\begin{array}{l}\text { RT } \\
\text { RT } \\
\text { RT }\end{array}$ & $\begin{array}{l}\text { Brazil } \\
\text { Brazil } \\
\text { Brazil }\end{array}$ & $\begin{array}{c}{[134]} \\
{[52]} \\
{[135]}\end{array}$ \\
\hline 157 & $\begin{array}{l}\text { 7-[[6-O-(6-deoxy- } \alpha-L- \\
\text { mannopyranosyl)- } \beta-D- \\
\text { glucopyranosyl]oxy]- } \\
\text { 5-hydroxy-2-(4- } \\
\text { hydroxy-3-methoxy- } \\
\text { phenyl)-3-methoxy- } \\
\text { 4H-1-benzopyran-4- } \\
\text { one }\end{array}$ & $\begin{array}{l}\text { quercetin } 3,3 \text { '- } \\
\text { dimethyl ether } 7-O- \\
\alpha-L- \\
\text { rhamnopyranosyl- } \\
(1 \rightarrow 6)-\beta-D- \\
\text { glucopyranoside }\end{array}$ & $\begin{array}{l}\text { RT } \\
\text { RT }\end{array}$ & $\begin{array}{l}\text { Brazil } \\
\text { Brazil }\end{array}$ & $\begin{array}{c}{[134]} \\
{[52]}\end{array}$ \\
\hline 158 & $\begin{array}{l}\text { 7-[[6-O-(6-deoxy- } \alpha-L- \\
\text { mannopyranosyl)- } \beta \text { - } D \text { - } \\
\text { glucopyranosyl]oxy]- } \\
5 \text {-hydroxy-2-(3- } \\
\text { hydroxy-4-methoxy- } \\
\text { phenyl)-3-methoxy- } \\
4 \text { H-1-benzopyran-4- } \\
\text { one }\end{array}$ & $\begin{array}{l}\text { quercetin } 3,4^{\prime}- \\
\text { dimethyl ether-7-O- } \\
\text { rutinoside }\end{array}$ & $\begin{array}{l}\text { AP } \\
\text { AP }\end{array}$ & $\begin{array}{l}\text { China } \\
\text { China }\end{array}$ & $\begin{array}{l}{[121]} \\
{[102]}\end{array}$ \\
\hline 159 & $\begin{array}{l}\text { 2-(3,4-dihydroxy- } \\
\text { phenyl)-3-( } \beta \text { - } D- \\
\text { glucofuranosyloxy)- } \\
5,7-\text { dihydroxy-4H-1- } \\
\text { benzopyran-4-one }\end{array}$ & isoquercitrin & $\begin{array}{l}\text { AP } \\
\text { AP }\end{array}$ & $\begin{array}{l}\text { Japan } \\
\text { China }\end{array}$ & $\begin{array}{c}{[83]} \\
{[102]}\end{array}$ \\
\hline \multicolumn{6}{|c|}{ Terpenoids } \\
\hline \multicolumn{6}{|c|}{ Sesquiterpenes } \\
\hline 160 & $\begin{array}{l}\text { 3,7,11,11-tetramethyl- } \\
\text { bicyclo[8.1.0]undeca- } \\
\text { 2,6-diene }\end{array}$ & bicyclogermacrene & LF & Brazil & [46] \\
\hline
\end{tabular}


Table 1. Cont.

\begin{tabular}{|c|c|c|c|c|c|}
\hline 161 & $\begin{array}{l}\text { 4,11,11-trimethyl-8- } \\
\text { methylenebicyclo- } \\
\text { [7.2.0]undec-4-ene }\end{array}$ & $E$-caryophyllene & LF & Brazil & {$[46]$} \\
\hline 162 & $\begin{array}{l}\text { 1-methyl-5-methylene- } \\
\text { 8-(1-methylethyl)-1,6- } \\
\text { cyclodecadiene }\end{array}$ & germacrene-D & LF & Brazil & {$[46]$} \\
\hline 163 & $\begin{array}{l}\text { 4-(1,5-dimethyl-4- } \\
\text { hexen-1-ylidene)-1- } \\
\text { methyl-cyclohexene }\end{array}$ & $Z$ - $\gamma$-bisabolene & LF & Brazil & {$[46]$} \\
\hline 164 & $\begin{array}{l}\text { decahydro-1,1,4- } \\
\text { trimethyl-7-methylene- } \\
1 \mathrm{H} \text {-cycloprop[e]- } \\
\text { azulene }\end{array}$ & $\beta$-gurjunene & LF & Brazil & {$[46]$} \\
\hline 165 & $\begin{array}{l}\text { 2,6,6,9-tetramethyl- } \\
1,4,8 \text {-cycloundeca- } \\
\text { triene }\end{array}$ & $\begin{array}{l}\alpha \text {-humulene; } \\
\alpha \text {-caryophyllene }\end{array}$ & LF & Brazil & {$[46]$} \\
\hline 166 & & $\delta$-muurolene & LF & Brazil & {$[46]$} \\
\hline 167 & $\begin{array}{l}\text { 1,2,3,4,4a,5,6,8a- } \\
\text { octahydro-4a,8- } \\
\text { dimethyl-2-(1- } \\
\text { methylethylidene)- } \\
\text { naphthalene }\end{array}$ & $\begin{array}{l}\text { selina-3,7(11)- } \\
\text { diene }\end{array}$ & LF & Brazil & {$[46]$} \\
\hline \multicolumn{6}{|c|}{ Diterpenes } \\
\hline 168 & $\begin{array}{l}(2 E, 7 R, 11 R)-3,7,11,15- \\
\text { tetramethyl-2- } \\
\text { hexadecen-1-ol }\end{array}$ & phytol & EP & Taiwan & {$[85]$} \\
\hline 169 & $\begin{array}{l}\text { 3,7,11,15-tetramethyl- } \\
\text { 2-hexadecenoic acid }\end{array}$ & phytenic acid & EP & Taiwan & {$[85]$} \\
\hline 170 & $\begin{array}{l}\text { 3,7,11,15-tetramethyl- } \\
\text { 2-hexadecenyl ester- } \\
\text { heptanoic acid }\end{array}$ & phythyl heptanoate & LF & not stated & {$[84]$} \\
\hline \multicolumn{6}{|c|}{ Steroids } \\
\hline 171 & & campestrol & AP & Tanzania & {$[44]$} \\
\hline 172 & & phytosterin-B & $\begin{array}{l}\mathrm{NF} \\
\mathrm{NF}\end{array}$ & $\begin{array}{l}\text { Taiwan } \\
\text { Egypt }\end{array}$ & $\begin{array}{l}{[112]} \\
{[86]}\end{array}$ \\
\hline 173 & stigmast-5-en-3-ol & $\beta$-sitosterol & $\begin{array}{l}\mathrm{NF} \\
\mathrm{AP} \\
\mathrm{EP}\end{array}$ & $\begin{array}{l}\text { Taiwan } \\
\text { Tanzania } \\
\text { Taiwan }\end{array}$ & $\begin{array}{l}{[91]} \\
{[44]} \\
{[85]}\end{array}$ \\
\hline
\end{tabular}


Table 1. Cont.

174

$\beta$-sitosterol glucoside

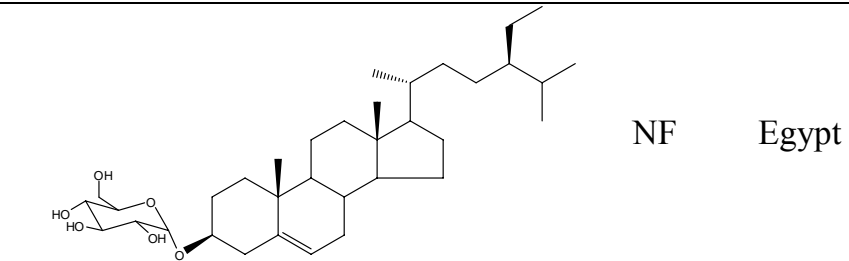

[86]

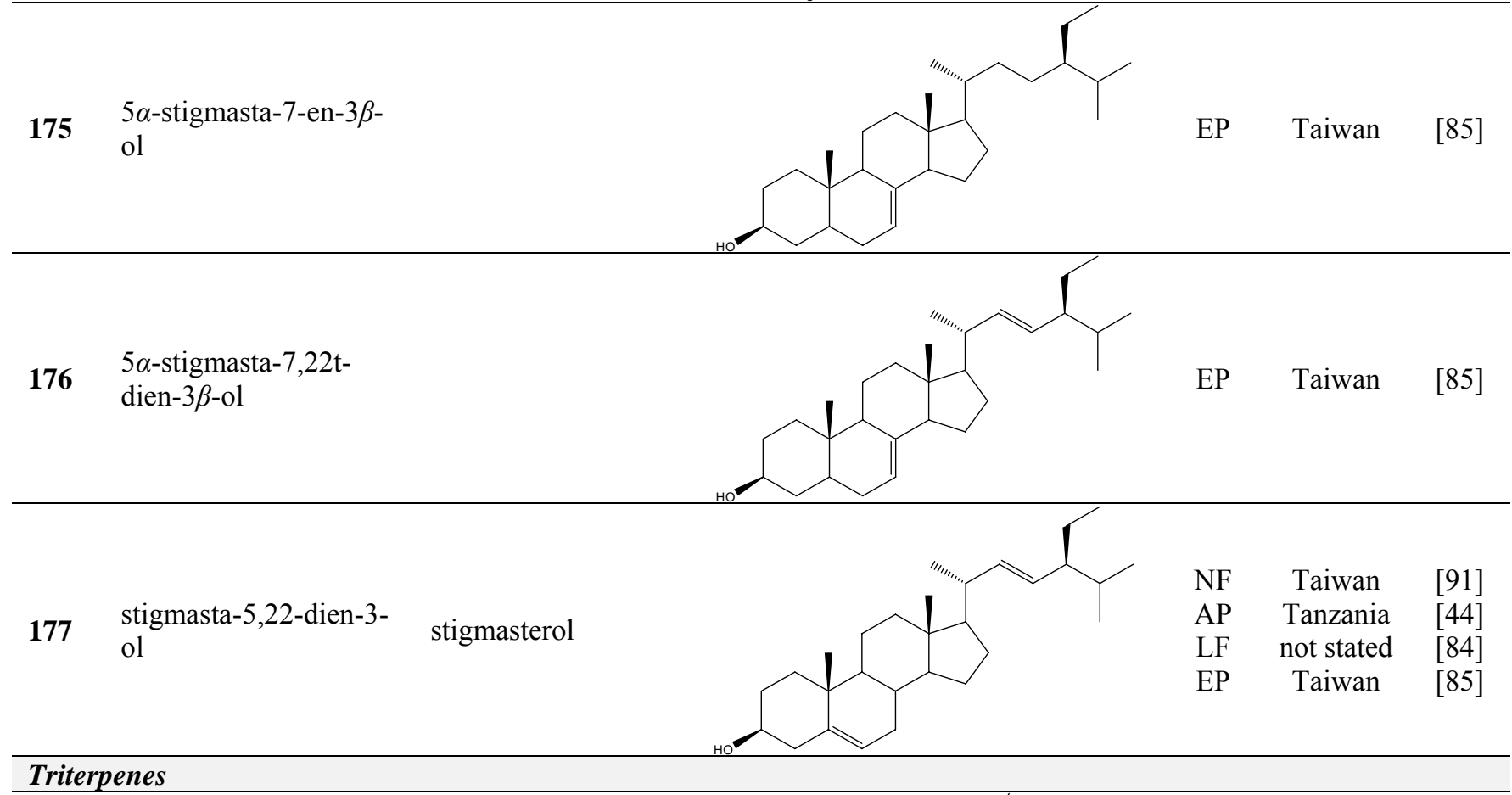

\section{Triterpenes}

178 lup-20(29)-en-3-ol lupeol<smiles>CC1CC[C@]2(C)CC[C@]3(C)C(CCC4[C@@]5(C)CC[C@H](O)C(C)(C)C5CC[C@]43C)C12</smiles>

NF Egypt

[86] $179 \begin{aligned} & \text { lup-20(29)-en-3-ol, lupeol acetate } \\ & \text { acetate }\end{aligned}$

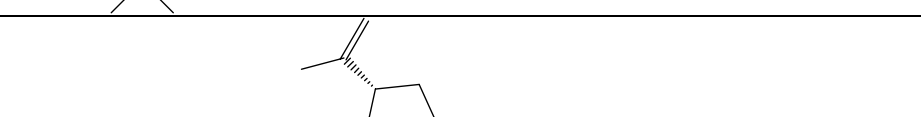<smiles>CC(=O)O[C@H]1CC[C@]2(C)[C@@H](CC[C@H]3C(C)CCC[C@]32C)C1(C)C</smiles>

NF Egypt

[86]

180 olean-12-en-3-ol

$\beta$-amirin

NF Egyp

[86]

5,9,13-trimethyl-

$18124,25,26-$

trinoroleanan-3-ol friedelan-3 $\beta$-ol

AP Tanzania

[44] 
Table 1. Cont.

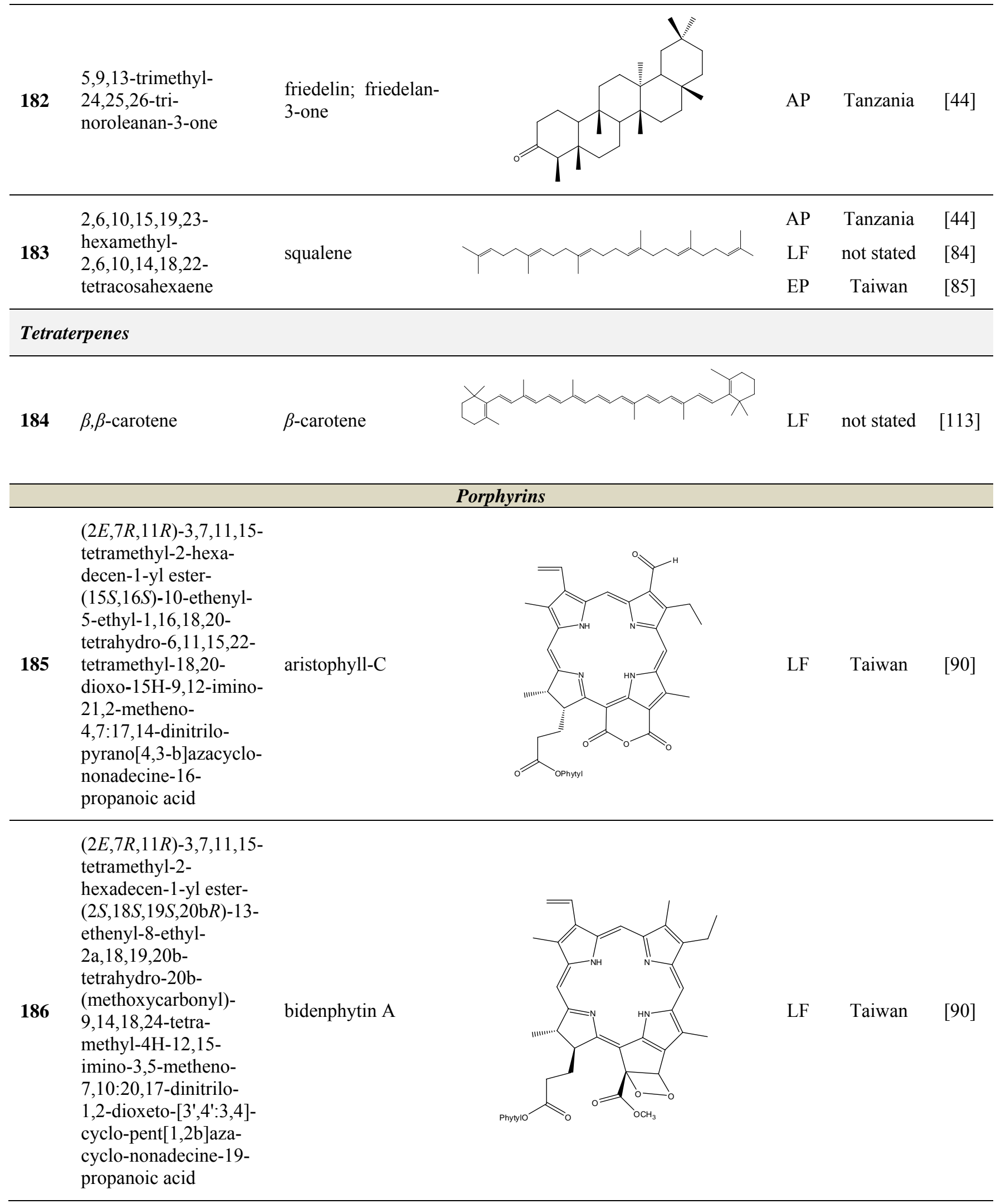


Table 1. Cont.

$(2 E, 7 R, 11 R)-3,7,11,15-$

tetramethyl-2-hexa-

decen-1-yl ester-

(2S,18S,19S,20bR)-13-

ethenyl-8-ethyl-

2a, 18,19,20b-

tetrahydro-2a-hydroxy-

187 20b-(methoxy-

carbonyl)-9,14,18,24-

bidenphytin B

tetramethyl-4H-12,15-

imino-3,5-metheno-

7,10:20,17-dinitrilo-

1,2-dioxeto[3',4':3,4]-

cyclo-pent[1,2-b]-

azacyclononadecine-

19-propanoic acid

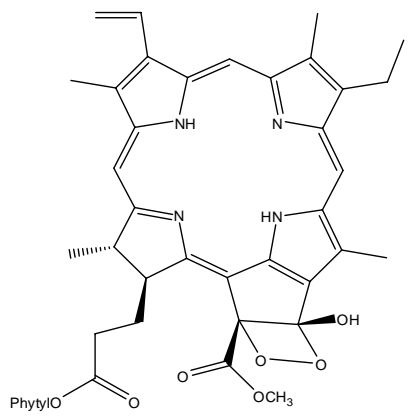

LF Taiwan

[90]

$(2 E, 7 R, 11 R)-3,7,11,15-$ tetramethyl-2-

hexadecen-1-yl ester$(3 R, 4 S, 21 R)$-14-ethyl-

188

21-hydroxy-21-

(methoxycarbonyl)-

4,8,9,13,18-penta-

methyl-20-oxo-3-

phorbinepropanoic

acid

$(2 E, 7 R, 11 R)-3,7,11,15-$

tetramethyl-2-

hexadecen-1-yl ester-

$(3 R, 4 S, 21 S)$-14-ethyl-

189

21-hydroxy-21-

(methoxycarbonyl)-

$4,8,9,13,18$ -

$\left(13^{2} R\right)-13^{2}-$

hydroxy-

pheophytin a

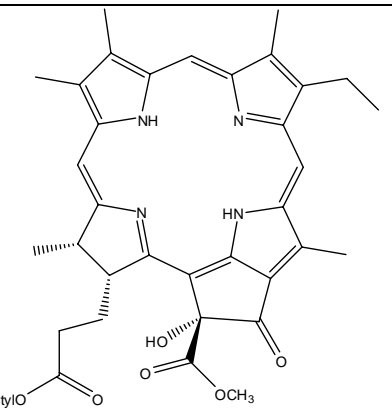

LF Taiwan

[90]

pentamethyl-20-oxo-3-

phorbinepropanoic

acid

$\left(13^{2} S\right)-13^{2}-$

hydroxy-

pheophytin a

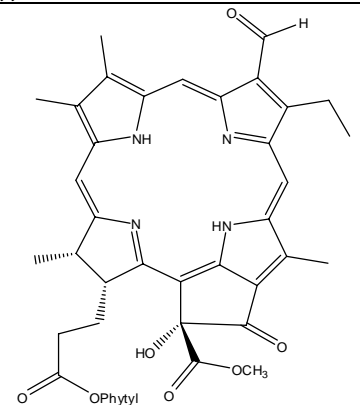

LF Taiwan

[90]

$(2 E, 7 R, 11 R)-3,7,11,15-$

tetramethyl-2-hexa-

decen-1-yl ester-

$(3 R, 4 S, 21 R)$-14-ethyl-

$\left(13^{2} R\right)-13^{2}-$

13-formyl-21-hydroxy-

21-(methoxycarbonyl)-

hydroxy-

4,8,9,18-tetramethyl-

pheophytin $b$

20-oxo-3-phorbine-

propanoic acid,

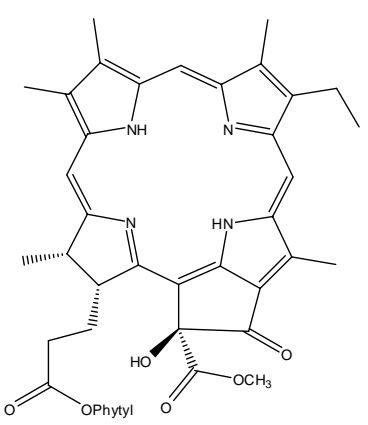

LF Taiwan

[90] 
Table 1. Cont.

$(2 E, 7 R, 11 R)-3,7,11,15-$ tetramethyl-2-

hexadecen-1-yl ester$(3 R, 4 S, 21 S)$-14-ethyl- $\quad\left(13^{2} S\right)-13^{2}-$

191 13-formyl-21-hydroxy- hydroxy-

21-(methoxycarbonyl)- pheophytin $b$ 4,8,9,18-tetramethyl20-oxo-3-phorbinepropanoic acid

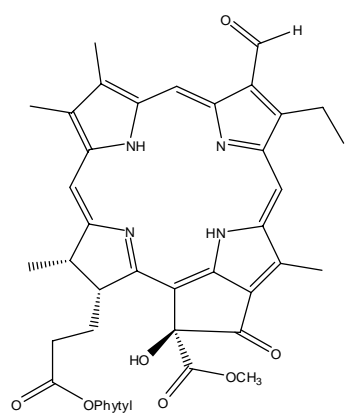

$(2 E, 7 R, 11 R)-3,7,11,15-$ tetramethyl-2-hexadecen-1-yl ester$(3 S, 4 S, 21 R)-9$-ethenyl-

192 14-ethyl-21-(methoxy- pheophytin a carbonyl)-4,8,13,18tetramethyl-20-oxo-3phorbinepropanoic acid

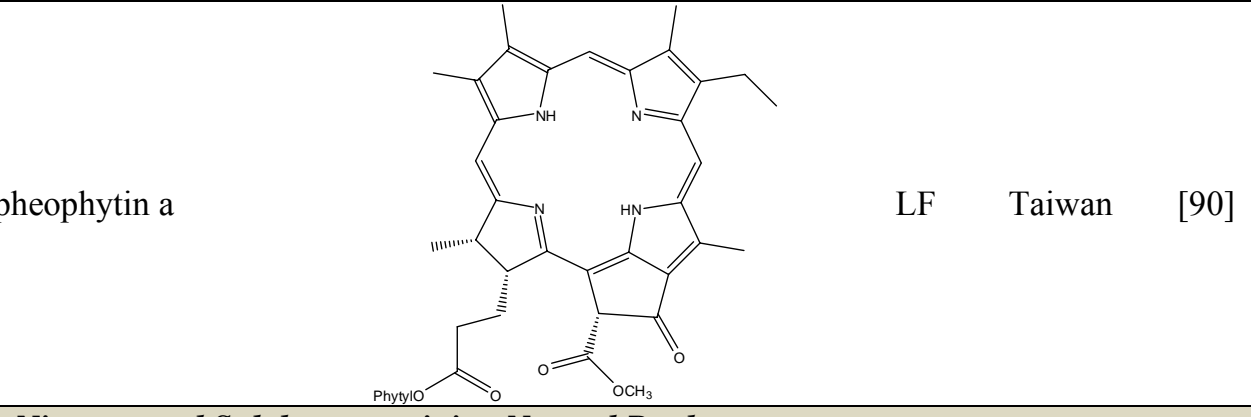

Nitrogen and Sulphur-containing Natural Products

3,7-dihydro-1,3,7-

193 trimethyl-1H-purine- caffeine 2,6-dione

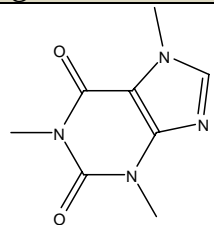

AP Uganda

[110]

(1)

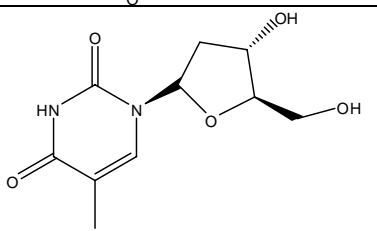

NF China

[99]

194 thymidine

195 1-(2-thienyl)-ethanone 2-acetyl-thiophene

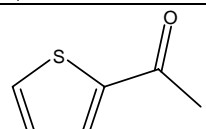

RT Germany

[122]

\section{Carbohydrates/ disaccharides}

heptanyl 2-O- $\beta$ -

xylofuranosyl-

$(1 \rightarrow 6)-\beta$ -

glucopyranoside

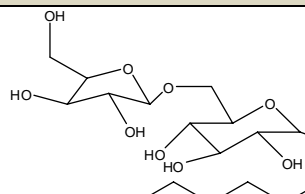

EP Taiwan

[79]

\section{Miscellaneous}

$2-[(3 R, 7 R, 11 R)-3-$

hydroxy-3,7,11,15-

197 tetramethylhexadecyl]-

3,5,6-trimethyl-2,5-

cyclohexadiene-1,4-

dione $\alpha$-tocopheryl

quinone

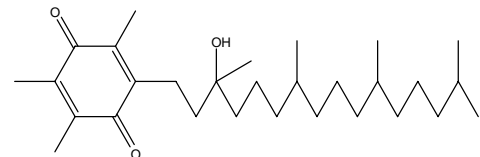

EP Taiwan

[85]

7-O-(4",6"'-

diacetyl)- $\beta-D$ -

glucopyranoside
NF

LF China

[59]

AP, Aerial part; LF, Leaf; ST, Steam; EP, Entire plant; FL, Flowers; RT, Root; SD, Seed; LTC, Leaves of tissue culture; PNS, Part not specified; NF, Not found. 


\section{Acetylene compounds}

The acetylenes are one class of aliphatic hydrocarbons that has a taxonomically interesting distribution pattern in higher plant families; they occur regularly in only five families, namely the Campanulaceae, Asteraceae, Araliaceae, Pittosporaceae and Umbelliferae [95]. Within the Asteraceae family, these compounds are widely distributed in the Heliantheae tribe [2,4]. The genus Bidens is known to produce compounds of this class [5]. They occur in all parts of the plant, often accumulating in roots [96].

To date 34 acetylenes (compounds 35-68) were isolated from B. pilosa (Table 1). The $\mathrm{C}_{13^{-}}$ polyacetylenes are the most abundant in the species and among them, ene-tetryn-ene 36 and its alcohol, acetyl and aldehyde oxygenated derivatives 40-42, $\mathrm{C}_{13}$-phenylacetylenes 59-66 and $\mathrm{C}_{13}$ acetylenes with an ene-triyn-diene chromophore 39 are typical constituents within the genus Bidens $[2,4,96,97]$.

The principal representative of the $\mathrm{C}_{13}$-polyacetylenes is 1-phenylhepta-1,3,5-triyne (64). This $\mathrm{C}_{13^{-}}$ phenylacetylene is abundant in $B$. pilosa and is present in leaves, stems and roots of the species $[5,73,96,97]$. The compound is biologically active and several studies have reported that it strongly absorbs long-wave UV radiation, and the activity is altered upon exposure to light (photo activation) [98].

The occurrence of $\mathrm{C}_{17}$-acetylenes is rare in the genus, being limited to the Hawaiian species of Bidens [4], while one compound (35) was related to B. pilosa grown in China [2,99]. Also, three $\mathrm{C}_{14^{-}}$ acetylenes 39,44,46, with one (46) being common in species of genus Coreopsis, and another (44), a new compound, were reported first in B. pilosa [4,51,100].

Another group of polyacetylenes isolated from B. pilosa are the polyacetylene glucosides (PAGs), which are glycosides of polyacetylenes in which a sugar moiety (glycose or rhamnose) is joined to a polyacetylene through an - $O$-glucosidic linkage. Of even more restricted distribution, these have been reported for only two families, Asteraceae and Campanulaceae. So far 22 PAGs are known, however most of them have been isolated from Bidens species [101].

Studies report the isolation of nine PAGs (50-58) from different parts from B. pilosa. Four compounds $(\mathbf{5 0}, \mathbf{5 3}-\mathbf{5 5})$ have the common $\mathrm{C}_{13}$-acetylene linkage to glycoside portion in the $\mathrm{C}_{2}$ position $[49,54,61,102]$, however the glycoside derivates of $\mathrm{C}_{14}$-acetylene have the linkage to the glycoside portion in the terminal portion (52) and C3 (51) [53,54]. Other unusual three PAGs have also been reported for $B$. pilosa. Two $\mathrm{C}_{16}$-acetylenes $\left(\mathbf{5 6 , 5 7 )}\right.$ and one $\mathrm{C}_{17}$-acetylene $(\mathbf{5 8})$ having an ester in the terminal portion linkage to a carboxylic acid [70].

Phenylthiophenes, classified as $\mathrm{C}_{13}$-acetylene and related compounds [4], are related to only occur in Coreopsis and in Hawaiian Bidens [4,103], however a phenylthiophene 67 and its glycosylate 68 were reported for B. pilosa growing in China [100].

\section{Flavonoids}

Flavonoids are the class of compound of higher occurrence in the species and are described as chemotaxonomic markers at lower hierarchical levels of the Asteraceae [104]. According to the Bidens genus, the flavonoid profile of B. pilosa is a complex one that includes aurones, chalcones, flavanones, 
flavones and flavonols with a wide variety of $O$-methylation patterns and glycosylations [105], totaling 58 different compounds isolated to date (Table 1).

Anthochlors (aurones and chalcones) are found in a number of plant families, including the Asteraceae. However research indicates that, despite some variations, anthochlors are good markers for the taxonomic subtribe Coreopsidinae (Heliantheae tribe), thus representing the only case in the family Asteraceae in which a certain type of flavonoid is taxonomically diagnostic at the sub tribal level [106].

Species of Bidens typically contain the chalcones butein (3,4,3',4'-tetrahydroxychalcone, 112), okanin $\left(3,4,2^{\prime}, 3^{\prime}, 4^{\prime}\right.$-pentahydroxychalcone, 113) and their 4'-glycosides [3]. Of the aurones, maritimetin $\left(6,7,3^{\prime}, 4^{\prime}\right.$-tetrahydroxyaurone, 102) and sulfuretin $\left(6,3^{\prime}, 4^{\prime}\right.$-tetrahydroxyaurone, 101) and their glycosides are commonly found in the genus [107]. These compounds have been reported for $B$. pilosa [108].

In B. pilosa, the glycosides aurones are frequent in position $6(\mathbf{1 0 3 - 1 1 0})$ and rare in 7 (104) while the glycosides derived from chalcones (111,114-128) are in the positions 3' and 4'. Two chalcone glycosides, one in position 2' (111) and other in 4 (128) were also found to the specie [59,102]. Most of these compounds are acylated with $p$-coumaric and/or acetic acid on the sugar moiety and are

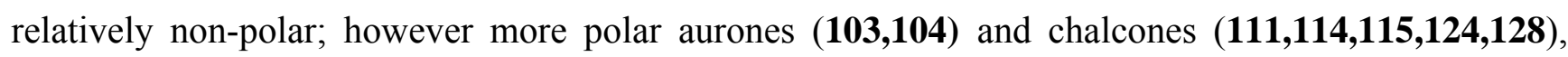
mono- and diglucosides were isolated from aerial parts [109]. Two B-ring methylated chalcones (126-127) [80,100] were also found in the species, but this kind of derivatives is rarely reported in the Bidens genus [3].

Flavones and flavonols identified from members of Bidens are for the most part commonly encountered compounds, i.e., glycosides of apigenin, luteolin, kaempferol and quercetin [105]. $B$. pilosa maintains that standard, however some flavonols present methoxy substitutent groups at their positions 3, 6, 7, 3' and/or 4', as in jacein (149), centaureidin (139) and its glycoside centaurein (140) [74,79]. Among the flavones 5-O-methylhoslundin (135) was reported, a compound previously isolated only from Hoslundia opposite (Lamiaceae) [110]. This unusual compound presents methoxy substituted groups in $\mathrm{C} 5$ and $\mathrm{C} 7$ and a pyranone derivative at $\mathrm{C} 6$.

\section{Other compound classes}

Several other compound classes have been isolated from different parts of B. pilosa and are listed in Table 1. Among these, aliphatic hydrocarbon derivatives and simple aromatic hydrocarbons have been reported, although these constituents are rather ubiquitous in plants. Long chain saturated unbranched hydrocarbons between $C_{21}$ and $C_{33}(\mathbf{1}-13)$ have been isolated of $B$. pilosa [44,91]. Of the saturated unbranched alcohols, the compound 2-butoxyethanol (14) is the only ether-ethanol, while for the unbranched aliphatic carboxylic acid and ester group, three compounds have ether-ester functions (3234). The simple aromatic hydrocarbons and simple phenylpropanoid compounds form two small groups of natural products in B. pilosa. In the first, vanillic (80), salicylic (78) and protocatechuic (79) acids and their derivatives are predominant [87], while the phenylpropanoids are represented by coumaric (82), ferulic (84) and caffeic (85) acid. In this group, one new disubstituted acetylacetone (86) was described for B. pilosa growing in India [90].

Also in the phenylpropanoids group, caffeoyl ester derivatives 87-97 are fairly reported for the specie, and some esters formed by the combination of two caffeic acids to one quinic acid (93-97) 
$[79,83]$ or one caffeic acid to one erythronic acid (88-92) [88]. The only coumarin (100) described for B. pilosa is usually found in other species of the family [86].

Of the mevalonate pathway, several sesquiterpenes (160-167), sterols (171-177) and triterpenes (178-183) have been isolated of leaves from B. pilosa $[44,51,86]$. The sesquiterpenes reported were characterized by GC-MS [46]. These are divided into mono- and bicyclic, commonly found in leaf extracts from Asteraceae. In the diterpenes, acyclic phytane diterpenoids have been reported; among them phytyl heptanoate (170) is an unusual compound that has an aliphatic chain of seven carbon atoms linked to the terminal acid portion [84].

The most abundant sterols from B. pilosa are stigmasterol (177) and sitosterol (173), which are ubiquitous compounds of plant cell membranes [111]. Stigmasterol derivates $(\mathbf{1 7 5 , 1 7 6 )}$, sitosterol glucoside (174) [85,91] and phytosterin B (172), a phytosterin first isolated in B. pilosa [112] has also been reported. Among the triterpenes, only squalene (183) is an acyclic one. The friedelanes 181,182 and lupeol derivatives 178, 179 are the more common triterpenes reported for B. pilosa $[44,86]$. Among the tetraterpenes $\beta$-carotene (184) is reported to be present in high concentration in young leaves of B. pilosa [113].

Chlorin (=2,3-dihydroporphyrin) and its derivatives - including chlorophyll, pheophytin, chlorophyllin, pheophobide, and many other closely related analogues - are found in most higher plants, algae, and even bacteria [114]. For B. pilosa two new pheophytins $(\mathbf{1 8 6 , 1 8 7})$, with peroxide functionalities in ring $E$ were reported, besides another six pheophytins (185,188-192), already known [114].

Only two representatives of the class of nitrogen-containing natural products, one being the nucleoside thymidine (194) are reported [122]. One thyophene (195) was reported from B. pilosa [99]. One disaccharide (196) was isolated from an entire B. pilosa. Also, two miscellaneous representatives were reported, a quinone linked to an aliphatic chain (197) [85] and one compound of unidentified structure (198) [59].

The content of essential oil from flowers, leaves and stems of B. pilosa has been analyzed by GCMS in China, Japan, USA, Cameroon, Nigeria and Iran [66,115-120,136]. In this review, the series of components identified as being commonly found in plants containing essential oils and present mostly in very small quantities are not listed. It is then just a brief comment about the main and unusual constituents. In the species a series of mono- and sesquiterpenes have been detected [66,116,117-119]. The major constituents are the sesquiterpenes germacrene-D and $\beta$-caryophyllene. Polyacetylenes (36,59,60,64), including 1-phenylhepta-1,3,5-tryin (64) have been identified in root oil and aerial parts $[117,119]$. A chromone, known as precocene I, isolated from oil of the leaves from B. pilosa also was reported [116].

\section{Acknowledgements}

The authors are grateful to $\mathrm{CNPq} / \mathrm{RENORBIO}$ and CAPES/Brazil for financial support and research fellowships. 


\section{References}

1. Karis, P.O.; Ryding, O. Asteraceae: Cladistics and Classification; Bremer, K., Ed.; Tember Press: Portland, OR, USA, 1994; pp. 559-569.

2. Bohlmann, F.; Burkhardt, T.; Zdero, C. Naturally Occurring Acetylenes; Academic Press: New York, NY, USA, 1973; pp. 356-359.

3. McCormick, S.P.; Bohm, B.A.; Ganders, F.R. Methylated chalcones from Bidens torta. Phytochemistry 1984, 23, 2400-2401.

4. Christensen, L.P.; Lam, J. Acetylenes and related compounds in Heliantheae. Phytochemistry 1991, 30, 11-49.

5. Jensen, S.L.; Sörensen, N.A. Studies related to naturally occurring acetylene compounds. XXIX. Preliminary investigations in the genus Bidens. I. Bidens radiate and Bidens ferulaefolia. Acta Chem. Scand. 1961, 15, 1885-1891.

6. Young, P.H.; Hsu, Y.-J.; Yang, W.-C. Bidens pilosa L. and its medicinal use. In Recent Progress in Medicinal Plants; Awaad, A.S., Singh, V.K., Govil, J.N., Eds.; Studium Press LLC: New Delhi, India, 2010; Volume 28, pp. 411-426.

7. Wang, Q.; Zhang, Y.-N.; Chen, F.-H. Research development in chemical constituents and pharmacological action of total flavonoids of Bidens bipinnata L. Anhui Yiyao. 2009, 13, 1011-1013.

8. Aridogan, B.C. Immunomodulatory effects of phytocompounds. In Modern Phytomedicine: Turning Medicinal Plants into Drugs; Ahmad, I., Aqil, F., Owais, M., Eds.; Wiley-VCH: Dublin, Ireland, 2006; pp. 341-356.

9. Guerra, F.; Goyos, C. Pharmacology of Mexican antidiabetic plants. III. Action of aceitilla, Bidens leucantha, on normal and diabetic blood sugar. Prensa Med. Mex. 1951, 16, 7-11.

10. Astudillo, V.A.; Davalos, V.H.; De Jesus, L.; Herrera, G.; Navarrete, A. Investigation of Alternanthera repens and Bidens odorata on gastrointestinal disease. Fitoterapia 2008, 79, 577-580.

11. Abraham, Z.; Bhakuni, S.D.; Garg, H.S.; Goel, A.K.; Mehrotra, B.N.; Patnaik, G.K. Screening of Indian plants for biological activity. Part XII. Indian J. Exp. Biol. 1986, 24, 48-68.

12. Leonard, D.B. Medicine at your Feet: Healing Plants of the Hawaiian Kingdom Bidens spp. (Kïnehi); Roast Duck Producktion: Kapaa-Princeville, HI, USA, 2006; pp. 1-15.

13. Moura, M.D.; Torres, A.R.; Oliveira, R.A.G.; Diniz, M.F.F.M.; Barbosa-Filho, J.M. Natural products as inhibitors of models of mammary neoplasia. Brit. J. Phytother. 2001, 5, 124-145.

14. Moura, M.D.; Silva, J.S.; Oliveira, R.A.G.; Diniz, M.F.F.M.; Barbosa-Filho, J.M. Natural products reported as potential inhibitors of uterine cervical neoplasia. Acta Farm. Bonaerense 2002, 21, 67-74.

15. Silva, J.S.; Moura, M.D.; Oliveira, R.A.G.; Diniz, M.F.F.M.; Barbosa-Filho, J.M. Natural product inhibitors of ovarian neoplasia. Phytomedicine 2003, 10, 221-232.

16. Gonçalves, M.C.R.; Moura, L.S.A.; Rabelo, L.A.; Barbosa-Filho J.M.; Cruz, H.M.M.; Cruz, J. Natural products inhibitors of HMG CoA reductase. Rev. Bras. Farm. 2000, 81, 63-71. 
17. Barbosa-Filho, J.M.; Medeiros, K.C.P.; Diniz, M.F.F.M.; Batista, L.M.; Athayde-Filho, P.F.; Silva, M.S.; Cunha, E.V.L.; Almeida, J.R.G.S.; Quintans-Júnior, L.J. Natural products inhibitors of the enzyme acetylcholinesterase. Rev. Bras. Farmacogn. 2006, 16, 258-285.

18. Barbosa-Filho, J.M.; Martins, V.K.M.; Rabelo, L.A.; Moura, M.D.; Silva, M.S.; Cunha, E.V.L.; Souza, M.F.V.; Almeida, R.N.; Medeiros, I.A. Natural products inhibitors of the angiotensin converting enzyme (ACE). A review between 1980-2000. Rev. Bras. Farmacogn. 2006, 16, 421-446.

19. Almeida, R.N.; Navarro, D.S.; Barbosa-Filho, J.M. Plants with central analgesic activity. Phytomedicine 2001, 8, 310-322.

20. Pereira, J.V.; Modesto-Filho, J.; Agra, M.F.; Barbosa-Filho, J.M. Plant and plant-derived compounds employed in prevention of the osteoporosis. Acta Farm. Bonaerense 2002, 21, 223-234.

21. Morais, L.C.S.L.; Barbosa-Filho, J.M.; Almeida, R.N.; Plants and bioactive compounds for the treatment of Parkinson's disease. Arq. Bras. Fitomed. Cientif. 2003, 1, 127-132.

22. Quintans-Júnior L.J.; Almeida, J.R.G.S.; Lima, J.T.; Nunes, X.P.; Siqueira, J.S.; Oliveira, L.E.G.; Almeida, R.N.; Athayde-Filho, P.F.; Barbosa-Filho, J.M. Plants with anticonvulsant properties - a review. Rev. Bras. Farmacogn. 2008, 18, 798-819.

23. Sousa, F.C.F.; Melo, C.T.V.; Citó, M.C.O.; Félix, F.H.C.; Vasconcelos, S.M.M.; Fonteles, M.M.F.; Barbosa-Filho, J.M.; Viana, G.S.B. Plantas medicinais e seus constituintes bioativos: Uma revisão da bioatividade e potenciais benefícios nos distúrbios da ansiedade em modelos animais. Rev. Bras. Farmacogn. 2008, 18, 642-654.

24. Rocha, L.G.; Almeida, J.R.G.S.; Macedo, R.O.; Barbosa-Filho, J.M. A review of natural products with antileishmanial activity. Phytomedicine 2005, 12, 514-535.

25. Amaral, F.M.M.; Ribeiro, M.N.S.; Barbosa-Filho, J.M.; Reis, A.S.; Nascimento, F.R.F.; Macedo, R.O. Plants and chemical constituents with giardicidal activity. Rev. Bras. Farmacogn. 2006, 16, 696-720.

26. Barbosa-Filho, J.M.; Nascimento-Júnior, F.A.; Tomaz, A.C.A.; Athayde-Filho, P.F.; Silva, M.S.; Cunha, E.V.L. Natural products with antileprotic activity. Rev. Bras. Farmacogn. 2007, 17, 141-148.

27. Barbosa-Filho, J.M.; Vasconcelos, T.H.C.; Alencar, A.A.; Batista, L.M.; Oliveira, R.A.G.; Guedes, D.N.; Falcão, H.S.; Moura, M.D.; Diniz, M.F.F.M.; Modesto-Filho, J. Plants and their active constituents from South, Central, and North America with hypoglycemic activity. Rev. Bras. Farmacogn. 2005, 15, 392-413.

28. Falcão, H.S.; Lima, I.O.; Santos, V.L.; Dantas, H.F.; Diniz, M.F.F.M.; Barbosa-Filho, J.M.; Batista, L.M. Review of the plants with anti-inflammatory activity studied in Brazil. Rev. Bras. Farmacogn. 2005, 15, 381-391.

29. Barbosa-Filho, J.M.; Piuvezam, M.R.; Moura, M.D.; Silva, M.S.; Lima, K.V.B.; Cunha E.V.L.; Fechine, I.M.; Takemura, O.S. Anti-inflammatory activity of alkaloids: a twenty century review. Rev. Bras. Farmacogn. 2006, 16, 109-139.

30. Mariath, I.R.; Falcão, H.S.; Barbosa-Filho, J.M.; Sousa, L.C.F.; Tomaz, A.C.A.; Batista, M.F.F.M.; Athayde-Filho, P.F.; Tavares, J.F.; Silva, M.S.; Cunha, E.V.L. Plants of the American continent with antimalarial activity. Rev. Bras. Farmacogn. 2009, 19, 158-192. 
31. Falcão, H.S.; Mariath, I.R.; Diniz, M.F.F.M.; Batista, L.M.; Barbosa- Filho, J.M. Plants of the American continent with antiulcer activity. Phytomedicine 2008, 15, 132-146.

32. Falcão, H.S.; Leite, J.A.; Barbosa-Filho, J.M.; Athayde-Filho, P.F.; Chaves, M.C.O.; Moura, M.D.; Ferreira, A.L.; Almeida, A.B.A.; Souza-Brito, A.R.M.; Diniz, M.F.F.M.; Batista, L.M. Gastric and duodenal antiulcer activity of alkaloids: a review. Molecules 2008, 13, 3198-3223.

33. Mota, K.S.L.; Dias, G.E.N.; Pinto, M.E.F.; Luiz-Ferreira, A.; Souza-Brito, A.R.M.; Hiruma-Lima, C.A.; Barbosa-Filho, J.M.; Batista, L.M. Flavonoids with gastroprotective activity. Molecules 2009, 14, 979-1012.

34. Ribeiro-Filho, J.; Falcão, H.S.; Batista, L.M.; Barbosa-Filho, J.M.; Piuvezam, M.R. Effects of plant extracts on HIV-1 protease. Curr. HIV Res. 2010, 8 , in press.

35. Agra, M.F.; França, P.F.; Barbosa-Filho, J.M. Synopsis of the plants known as medicinal and poisonous in Northeast of Brazil. Rev Bras Farmacogn. 2007, 17, 114-140.

36. Agra, M.F.; Silva, K.N.; Basílio, I.J.L.D.; França, P.F.; Barbosa-Filho, J.M. Survey of medicinal plants used in the region Northeast of Brazil. Rev. Bras Farmacogn. 2008, 18, 472-508.

37. Alves, J.S.; Castro, J.C.; Freire, M.O.; Cunha, E.V.L.; Barbosa-Filho, J.M.; Silva, M.S. Complete assignment of the $1 \mathrm{H}$ and $13 \mathrm{C}$ spectra of four triterpenes of the ursane, artane, lupane and friedelane groups. Magn. Reson. Chem. 2000, 38, 201-206.

38. Andrade, N.C.; Cunha, E.V.L.; Silva, M.S.; Agra, M.F.; Barbosa-Filho, J.M. Terpenoids of the Annonaceae: Distribution and compilation of 13C NMR data. In Recent Research Developments in Phytochemistry; Gayathri, A., Ed.; Research Signpost: Kerala, India, 2003; Volume 7, pp. 1-85.

39. Barbosa-Filho, J.M.; Sette, I.M.F.; Cunha, E.V.L.; Guedes, D.N.; Silva, M.S. Protoberberine alkaloids. In The Alkaloids; Cordell, G.A., Ed.; Elsevier Ltd.: London, UK, 2005; Volume 62, pp. $1-75$.

40. Barbosa-Filho, J.M.; Alencar, A.A.; Nunes, X.P.; Tomaz, A.C.A.; Sena-Filho, J.G.; AthaydeFilho, P.F.; Silva, M.S.; Souza, M.F.V.; Cunha, E.V.L. Sources of alpha-, beta-, gamma-, deltaand epsilon-carotenes: A twentieth century review. Rev. Bras. Farmacogn. 2008, 18, 135-154.

41. Sena-Filho, J.G.; Duringer, J.M.; Maia, G.L.A.; Tavares, J.F.; Xavier, H.S.; Silva, M.S.; Cunha, E.V.L.; Barbosa-Filho, J.M. Ecdysteroids from Vitex species: Distribution and compilation of their 13C-NMR spectral data. Chem. Biodivers. 2008, 5, 707-713.

42. Vasconcelos, S.M.M.; Honório-Júnior, J.E.R.; Abreu, R.N.D.C.; Silva, M.C.C.; Barbosa-Filho, J.M.; Lobato, R.F.G. Pharmacologic study of some plant species from the Brazilian Northeast: Calotropis procera, Agava sisalana, Solanum paludosum, Dioscorea cayenensis and Crotalaria retusa. In Medicinal Plants: Classification, Biosynthesis and Pharmacology, 4rd ed.; Varela, A., Ibañez, J., Eds.; Nova Science Publishers, Inc.: New York, NY, USA, 2009; pp. 189-202.

43. Oliveira, S.L.; Silva, M.S.; Tavares, J.F.; Sena-Filho, J.G.; Lucena, H.F.S.; Romero, M.A.V.; Barbosa-Filho, J.M. Tropane alkaloids from genus Erythrorylum: Distribution and compilation of C-NMR spectral data. Chem. Biodivers. 2010, 7, 302-326.

44. Geissberger, P.; Séquin, U. Constituents of Bidens pilosa L.: Do the components found so far explain the use of this plant in traditional medicine? Acta Tropica 1991, 48, 251-261.

45. Alvarez, A.; Pomar, F.; Sevilla, M.A.; Montero, M.J. Gastric antisecretory and antiulcer activities of an ethanolic extract of Bidens pilosa L. var. radiata Schult. Bip. J. Ethnopharmacol. 1999, 67, 333-340. 
46. Grombone-Guaratini, M.T.; Silva-Brandão, K.L.; Solferini, V.N.; Semir, J.; Trigo, J.R. Sesquiterpene and polyacetylene profile of Bidens pilosa complex (Asteraceae: Heliantheae) from southeast of Brazil. Biochem. Syst. Ecol. 2005, 33, 479-486.

47. Longuefosse, J.-L.; Nossin, E. Medical ethnobotany survey in Martinique I. J. Ethnopharmacol. 1996, 53, 117-142.

48. Rabe, T.; van Staden, J. Antibacterial activity of South African plants used for medicinal purposes. J. Ethnopharmacol. 1997, 56, 81-87.

49. Chiang, Y.-M.; Chang, C.L.-T.; Chang, S.-L.; Yang, W.-C.; Shyur, L.-F. Cytopiloyne, a novel polyacetylenic glucoside from Bidens pilosa, functions as a $\mathrm{T}$ helper cell modulator. $J$. Ethnopharmacol. 2007, 110, 532-538.

50. Brandão, M.G. L.; Krettli, A.U.; Soares, L.S.R.; Nery, C.G.C.; Marinuzzi, H.C. Antimalarial activity of extracts and fractions from Bidens pilosa and other Bidens species (Asteraceae) correlated with the presence of acetylene and flavonoid compounds. J. Ethnopharmacol. 1997, 57, 131-138.

51. Valdés, H.A.L.; Rego, H.P.L. Bidens pilosa Linné. Rev. Cubana Plant. Med. 2001, 1, 28-33.

52. Krettli, A.U.; Andrade-Neto, V.F.; Brandão, M.G.L.; Ferrari, W.M.S. The seach for new antimalarial drugs from plants used to treat fever and malaria or plants ramdomly selected: A review. Mem. I. Oswaldo Cruz. 2001, 96, 1033-1042.

53. Alvarez, L.; Marquina, S.; Villarreal, M.L.; Alonso, D.; Aranda, E.; Delgado, G. Bioactive polyacetylenes from Bidens pilosa. Planta Med. 1996, 62, 355-357.

54. Ubillas, R.P.; Mendez, C.D.; Jolad, S.D.; Luo, J.; King, S.R.; Carlson, T.J.; Fort, D.N. Antihyperglycemic acetylenic glucosides from Bidens pilosa. Planta Med. 2000, 66, 82-83.

55. Alarcon-Aguilar, F.J.; Roman-Ramos, R.; Flores-Saenz, J.L.; Aguirre-Garcia, F. Extracts of four mexican medicinal plants in normal and alloxan-diabetic mice. Phytother. Res. 2002, 16, 383-386.

56. Dimo, T.; Azay, J.; Tan, P.V.; Pellecuer, J.; Cros, G.; Bopelet, M.; Serrano, J.J. Effects of the aqueous and methylene chloride extracts of Bidens pilosa leaf on fructose-hypertensive rats. $J$. Ethnopharmacol. 2001, 76, 215-221.

57. Dimo, T.; Rakotonirina, S.V.; Tan, P.V.; Azay, J.; Dongo, E.; Cros, G. Leaf methanol extract of Bidens pilosa prevents and attenuates the hypertension induced by high-fructose diet in Wistar rats. J. Ethnopharmacol. 2002, 83, 183-191.

58. Leandre, K.K.; Claude, A.K.J.; Jacques, D.Y.; Flavien, T.; Etienne, E.E. $\beta$-Adrenomimetic actions in the hypotension and vasodilatation induced by a chromatographic active fraction from Bidens pilosa L. (Asteraceae) in mammals. Curr. Bioact. Compd. 2008, 4, 1-5.

59. Yuan, L.-P.; Chen, F.-H.; Ling, L.; Dou, P.-F.; Bo, H.; Zhong, M. M.; Xia, L.-J. Protective effects of total flavonoids of Bidens pilosa L. (TFB) on animal liver injury and liver fibrosis. $J$. Ethnopharmacol. 2008, 116, 539-546.

60. Parimalakrishnan, S.; Akalanka, D.; Anton, S; Gana, D. A.; Manavalan, R.; Sridhar, N. Studies of anticancer and antipyretic activity of Bidens pilosa whole plant. Afr. Health Sci. 2006, 6, 27-30.

61. Pereira, R.L.C.; Ibrahim, T.; Lucchetti, L.; Silva, A.J.R.; Moraes, V.L. G. Immunosuppressive and anti-inflammatory effects of methanolic extract and the polyacetylene isolated from Bidens pilosa L. Immunopharmacology 1999, 43, 31-37. 
62. Horiuchi, M; Seyama, Y. Improvement of the antiinflamatory and antiallergic activity of Bidens pilosa L. var. radiata Scherff treated with enzyme (cellulosine). J. Health Sci. 2008, 54, 294-301.

63. Chang, J.-S.; Chiang, L.-C.; Chen, C.-C.; Liu, L.-T.; Wang, K.-C.; Lin, C.-C. Antileukemic activity of Bidens pilosa L. var. minor (Blume) Sherff and Houttuynia cordata Thunb. Am. J. Chin. Med. 2001, 29, 303-312.

64. Rojas, J.J.; Ochoa, V.J.; Ocampo, S.A.; Munoz, J.F. Screening for antimicrobial activity of ten medicinal plants used in Colombian folkloric medicine: a possible alternative in the treatment of non-nosocomial infections. BMC Compl. Alternative Med. 2006, 6, 2.

65. Yang, H.-L.; Chen, S.-C.; Chang, N.-W.; Chang, J.-M.; Lee, M.-L.; Tsai, P.-C.; Fu, H.-H.; Kao, W.-W.; Chiang, H.-C.; Wang, H.H.; Hseu, Y.-C. Protection from oxidative damage using Bidens pilosa extracts in normal human erythrocytes. Food Chem. Toxicol. 2006, 44, 1513-1521.

66. Deba, F.; Xuan, T.D.; Yasuda, M.; Tawata, S. Chemical composition and antioxidant, antibacterial and antifungal activities of the essential oils from Bidens pilosa Linn. var. radiata. Food Control 2008, 19, 346-352.

67. Kviecinski, M.R.; Felipe, K.B.; Schoenfelder, T.; Wiese, L.P.L.; Rossi, M.H.; Gonçalez, E.; Felicio, J.D.; Filho, D.W.; Pedrosa, R.C. Study of the antitumor potential of Bidens pilosa (Asteraceae) used in Brazilian folk medicine. J. Ethnopharmacol. 2008, 117, 69-75.

68. Ministério da Saúde. RENISUS - Relação nacional de plantas medicinais de interesse ao SUS. http://portal.saude.gov.br/portal/arquivos/pdf/RENISUS.pdf (Accessed on 30 October 2010).

69. Pereira, R.L.C.; Ibrahim, T.; Lucchetti, L.; Silva, A.J.R.; Moraes, V.L.G. Immunosuppressive and anti-inflammatory effects of methanolic extract and the polyacetylene isolated from Bidens pilosa L. Immunopharmacology 1999, 43, 31-37.

70. Bairwa, K.; Kumar, R.; Sharma, R.J.; Roy, R.K. An updated review on Bidens Pilosa L. Pharma Chem. 2010, 2, 325-337.

71. Tobinaga, S.; Sharma, M.K.; Aalbersberg, W.G.L.; Watanabe, K.; Iguchi, K.; Narui, K.; Sasatsu, M.; Waki, S. Isolation and identification of a potent antimalarial and antibacterial polyacetylene from Bidens pilosa. Planta Med. 2009, 75, 624-628.

72. Kumari, P.; Misra, K.; Sisodia, B.S.; Faridi, U.; Srivastava, S.; Luqman, S.; Darokar, M.P.; Negi, A.S.; Gupta, M.M.; Singh, S.C.; Kumar, J.K. A promising anticancer and antimalarial component from the leaves of Bidens pilosa. Planta Med. 2009, 75, 59-61.

73. N’Dounga, M.; Balansard, G.; Babadjamian, A.; David, P.T.; Gasquet, M.; Boudon, G. Contribution a l'etude de Bidens pilosa L. identification et activite antiparasitaire de la phenyl-1 heptatriyne-1,3,5. Plant. Med. Phytother. 1983, 17, 65-75.

74. Chang, S.-L.; Chiang, Y.-M.; Chang, C. L.-T.; Yeh, H.-H.; Shyur, L.-F.; Kuo, Y.-H.; Wu, T.-K.; Yang, W.-C. Flavonoids, centaurein and centaureidin, from Bidens pilosa, stimulate IFNexpression. J. Ethnopharmacol. 2007, 112, 232-236.

75. Chang, S.-L.; Chang, C.L.-T.; Chiang, Y.-M.; Hsieh, R.-H.; Tzeng, C.-R.; Wu, T.-K.; Sytwu, H.K.; Shyur, L.-F.; Yang, W.-C. Polyacetylenic compounds and butanol fraction from Bidens pilosa can modulate the differentiation of helper $\mathrm{T}$ cells and prevent autoimmune diabetes in non-obese diabetic mice. Planta Med. 2004, 70, 1045-1051.

76. Yang, W.L.; Chang, L.; Yang, W. Treating type II diabetes involves administering to subject polyacetylenic compound or a Bidens pilosa preparation obtained by stirring pulverized Bidens 
pilosa in water at specific temperature to form suspension; and collecting supernatant. EP Patent 1955701 A1, 2008.

77. Wu, L.; Chiang, Y.; Chuang, H.; Wang, S.; Yang, G.; Chen, Y.; Lai, L.; Shyur, L.-F., Polyacetylenes function as anti-angiogenic agents. Pharm. Res. 2004, 21, 2112-2119.

78. Wu, L.-W.; Chiang, Y.-M.; Chuang, H.-C.; Lo, C.-P.; Yang, K.-Y.; Wang, S.-Y.; Shyur, L.-F. A novel polyacetylene significantly inhibits angiogenesis and promotes apoptosis in human endothelial cells through activation of the CDK inhibitors and caspase-7. Planta Med. 2007, 73, 655-661.

79. Chiang, Y.; Chuang, D.; Wang, S.; Kuo, Y.; Tsai, P.; Shyur, L. Metabolite profiling and chemopreventive bioactivity of plant extracts from Bidens pilosa. J. Ethnopharmacol. 2004, 95, 409-419.

80. Graham, K.; Graham, E.A.; Towers, G.H.N. Cercaricidal activity of phenylheptatriyne and $\alpha$ terthienyl, naturally occurring compounds in species of Asteraceae (Compositae). Can. J. Zool. 1980, 58, 1955-1958.

81. Christensen, L.P.; Lam, J.; Thomasen, T.A. A chalcone and other constituents of Bidens tripartitus. Phytochemistry 1990, 29, 3155-3156.

82. Chang, C.L.-T.; Chang, S.-L.; Lee, Y.-M.; Chiang, Y.-M.; Chuang, D.-Y.; Kuo, H.-K.; Yang, W.C. Cytopiloyne, a poliacetylenic glucoside, prevents type 1 diabetes in non-obese diabetic mice. $J$. Immunol. 2007, 178, 6984-6993.

83. Kusano, A.; Seyama, Y.; Usami, E.; Katayose, T.; Shibano, M.; Tsukamoto, D.; Kusano, G. Studies on the antioxidant active constituents of the dried powder from Bidens pilosa L. var. radiata Sch. Nat. Med. 2003, 57, 100-104.

84. Zulueta, M.C.A.; Tada, M.; Ragasa, C.Y. A diterpene from Bidens pilosa. Phytochemistry 1995, $38,1449-1450$.

85. Chang, M.-H.; Wang, G.-J.; Kuo, Y.-H.; Lee, C.-K. The low polar constituents from Bidens pilosa L. var. minor (Blume) Sherff. J. Chin. Chem. Soc. 2000, 47, 1131-1136.

86. Sarg, T.M.; Ateya, A.M.; Farrag, N.M.; Abbas, F.A. Constituents and biological activity of Bidens pilosa L. grown in Egypt. Acta Pharm. Hung. 1991, 61, 317-323.

87. Deba, F.; Xuan, T.D.; Yasuda, M.; Tawata, S. Herbicidal and fungicidal activities and identification of potential phytotoxins from Bidens pilosa L. var. radiata Scherff. Weed Biol. Manag. 2007, 7, 77-83.

88. Ogawa, K.; Sashida, Y. Caffeoyl derivatives of a sugar lactone and its hydroxy acid from the leaves of Bidens pilosa. Phytochemistry 1992, 31, 3657-3658.

89. Sashida, Y.; Ogawa, K.; Kitada, M.; Karikome, H.; Mimaki, Y.; Shimomura, H., New aurone glucosides and new phenylpropanoid glucosides form Bidens pilosa. Chem. Pharm. Bull. 1991, 39, 709-711.

90. Kumar, J.K.; Sinha, A.K. A new disubstituted acetylacetone from the leaves of Bidens pilosa Linn. Nat. Prod. Res. 2003, 17, 71-74.

91. Chen, A.H.; Lin, S.R.; Hong, C.H. Phytochemical study on Bidens pilosa L. var. Minor. Huaxue. 1975, 38-42.

92. Potawale, S.E.; Shinde, V.M.; Harle, U.N.; Borade, S.B.; Libi A.; Dhalawat, H.J.; Deshmukh, R.S. Bidens pilosa L.: a comprehensive review. Pharmacol. Online 2008, 2, 185-196. 
93. Bairwa, K.; Kumar, R.; Sharma, R.J.; Roy, R.K. An updated review on Bidens pilosa L. Der Pharma Chemica. 2010, 2, 325-337.

94. Buckingham, J. Introduction to the Type of Compound Index. In Dictionary of Natural Products; Chapman and Hall: London, UK, 1994; Volumes 6 and 7.

95. Harborne, J.B.; Baxter, H.; Moss, G.P. Phytochemical Dictionary: A Handbook of Bioactive Compounds from Plants; Taylor \& Francis: Philadelphia, PA, USA, 1999; Chapter 8, p. 57.

96. Towers, G.H.N.; Wat, C.-K. Biological activity of polyacetylenes. Rev. Latinoamer. Quim. 1978, 9, 162-170.

97. Wat, C.-K.; Biswas, R.K.; Graham, E.A.; Bohm, L.; Towers, G.H.N. Ultraviolet-mediated cytotoxic activity of phenylheptatriyne from Bidens pilosa L. J. Nat. Prod. 1979, 42, 103-111.

98. Wat, C.-K.; Johns, T.; Towers, G.H.N. Phototoxic and antibiotic activities of plants of the Asteraceae used in folk medicine. J. Ethnopharmacol. 1980, 2, 279-290.

99. Wang, S.; Yang, B.; Li, L.; Zhu, D.; He, D.; Wang, L. Active components of Bidens pilosa L. Zhongcaoyao 2005, 36, 20-21.

100. Wang, R.; Wu, Q.-X.; Shi, Y.-P. Polyacetylenes and flavonoids from the aerial parts of Bidens pilosa. Planta Med. 2010, 76, 893-896.

101. Ganjewalaa, D.; Kumara, S.; Ambikaa, K.; Luthrab, R. Plant polyacetylenic glycosides occurrence, biosynthesis and biological activities. Pharmacol. Online 2008, 2, 113-131.

102. Zhao, A.; Zhao, Q.; Peng, L.; Zhang, J.; Lin, Z.; Sun, H. A new chalcone glycoside from Bidens pilosa. Yunnan Zhiwu Yanjiu 2004, 26, 121-126.

103. Marchant, Y.Y.; Ganders, F.R.; Wat, C.K.; Towers, G.H. N. Polyacetylenes in Hawaiian Bidens. Biochem. Syst. Ecol. 1984, 12, 67-78.

104. Emerenciano, V.P .; Militão, J.S.L.T.; Campos, C.C.; Romoff, P.; Kaplan, M.A.C.; Zambon, M.; Brant, A.J.C. Flavonoids as chemotaxonomic markers for Asteraceae. Biochem. Syst. Ecol. 2001, 29, 947-957.

105. Bohman, B.A.; Stuessy, T.F. Flavonoids of the Sunflower Family (Asteraceae); Springer-Verlag: Wien, Austria, 2001; Chapter 11, pp. 260-263.

106. Crawford, D.J.; Stuessy, T.F. The taxonomic significance of anthochlors in the subtribe Coreopsidinae (Compositae, Heliantheae). Am. J. Bot.1981, 68, 107-117.

107. Isakova, T.I.; Serbin, A.G.; Belikov, V.V.; Chushenko, V.N. Flavonoids and polysaccharides of Bidens L. species. Rast. Resur. 1986, 22, 517-523.

108. Redl, K. Davis, B.; Bauer, R. Chalcone glycosides from Bidens campylotheca. Phytochemistry 1993, 32, 218-220.

109. Hoffmann, B.; Hölzl, J. Chalcone glucosides from Bidens pilosa. Phytochemistry 1989, 28, 247-249.

110. Sarker, S.D.; Bartholomew, B.; Nash, R.J.; Robinson, N. 5-O-methylhoslundin: an unusual flavonoid from Bidens pilosa (Asteraceae). Biochem. Syst. Ecol. 2000, 28, 591-593.

111. Brown, G.D. The biosynthesis of artemisinin (Qinghaosu) and the phytochemistry of Artemisia annua L. (Qinghao). Molecules 2010, 15, 7603-7698.

112. Lin, L.-L.; Wu, C.-Y.; Hsiu, H.-C.; Wang, M.-T.; Chuang, H. Diabetes mellitus. I. Hypoglycemic activity of phytosterin on alloxan-diabetic rats. Taiwan Yixuehui Zazhi 1967, 66, 58-66. 
113. Benhura, M.A.N.; Chitsiku, I.C. The extractable $\beta$-carotene content of Guku (Bidens pilosa) leaves after cooking, drying and storage. Int. J. Food Sci. Technol. 1997, 32, 495-500.

114. Lee, T.-H.; Lu, C.-K.; Kuo, Y.-H.; Jir-Mehng Lo, J.-M.; Lee, C.-K. Unexpected novel pheophytin peroxides from the leaves of Bidens pilosa. Helv. Chim. Acta 2008, 91, 79-84.

115. Sakuda, Y. Constituents of essential oils from Bidens pilosa L. and Ambrosia trifida L. Kochi Joshi Daigaku Kiyo, Shizen Kagakuhen 1988, 36, 1-5.

116. Zollo, P.H.A.; Kuiate, J.R.; Menut, C.; Lamaty, G.; Bessiere, J.M.; Chalchat, J.C.; Garry, R. Aromatic plants of tropical central Africa. Part XX. The occurence of 1-phenylhepta-1,3,5-triyne in the essential oil of Bidens pilosa L. from Cameroon. Flavour Frag. J. 1995, 10, 97-100.

117. Qin, J.; Chen, T.; Chen, S.; Lu, Q. Analysis of essential oil of Bidens pilosa L. by GC-MS. Fenxi Ceshi Xuebao 2003, 22, 85-87.

118. Dong L.; Yang, J.; Wang, X. Analysis of components of volatile oil from Bidens pilosa. Xinxiang Yixueyuan Xuebao 2004, 21, 179-180.

119. Priestap, H.A.; Bennett, B.C.; Quirke, J.M.E. Investigation of the essential oils of Bidens pilosa var. minor, Bidens alba and Flaveria linearis. J. Essent. Oil Res. 2008, 20, 396-402.

120. Riahi, S.; Ganjali, M.R.; Pourbasheer, E.; Norouzi, P. QSRR study of GC retention indices of essential - oil compounds by multiple linear regression with a genetic algorithm. Chromatographia. 2008, 67, 917-922.

121. Wang, J.; Yang, H.; Lin, Z.W.; Sun, H.D. Flavonoids from Bidens pilosa var. radiata. Phytochemistry 1997, 46, 1275-1278.

122. Bohlmann, F.; Bornowski, H.; Kleine, K.M. New polyynes from the tribe Heliantheae. Chem. Ber. 1964, 97, 2135-2138.

123. Chang, C.L.-T.; Kuo, H.-K.; Chang, S.-L.; Chiang, Y.-M.; Lee, T.-H.; Wu, W.-M.; Shyur, L.F.; Yang, W.-C. The distinct effects of a butanol fraction of Bidens pilosa plant extract on the development of Th1-mediated diabetes and Th2-mediated airway inflammation in mice. $J$. Biomed. Sci. 2005, 12, 79-89.

124. Chien, S.-C.; Young, P.H.; Hsu, Y.-J.; Chen, C.-H.; Tien, Y.-J.; Shiu, S.-Y.; Li, T.-H.; Yang, C.W.; Marimuthu, P.; Tsai, L.F.-L.; Yang, W.-C. Anti-diabetic properties of three common Bidens pilosa variants in Taiwan. Phytochemistry 2009, 70, 1246-1254.

125. Kusano, G.; Kusano, A.; Seyama, Y. Novel hypoglycemic and antiinflammatory polyacetylenic compounds, their compositions, Bidens plant extract fractions, and compositions containing the plant or the fraction. JP 2004083463 A, 2004.

126. Xia, Q.; Liu, Yuan; L., Y. Determination of gallic acid from different species and different medical parts of herba Bidens by RP-HPLC. Huaxi Yaoxue Zazhi 2009, 24, 308-310.

127. Chiang, Y.-M.; Lo, C.-P.; Chen, Y.-P.; Wang, S.-Y.; Yang, N.-S.; Kuo, Y.-H.; Shyur, L.-F. Ethyl caffeate suppresses NF- $\alpha$ B activation and its downstream inflammatory mediators, iNOS, COX2, and PGE2 in vitro or in mouse skin. Br. J. Pharmacol. 2005, 146, 352-363.

128. Hoffmann, B.; Hölzl, J. Acylated compounds from Bidens pilosa. Planta Med. 1989, 55, 108-109.

129. Hoffmann, B.; Hölzl, J. New chalcones from Bidens pilosa. Planta Med. 1988, 52-54.

130. Hoffmann, B.; Hölzl, J. A methylated chalcone glucoside from Bidens pilosa. Phytochemistry 1988, 27, 3700-3701. 
131. Hoffmann, B.; Hölzl, J. Weitere acylierte chalkone aus Bidens pilosa. Planta Med. 1988, 450-451.

132. Pham, V.V.; Pham, T.K.; Hoang, V.L.; Phan, V.K. Flavonoid compounds from the plant Bidens pilosa L. (Asteraceae). Tap Chi Duoc Hoc. 2010, 50, 48-53.

133. Xia, Q.; Liu, Y.; Li, Y. Determination of hyperoside in different parts and different species of herba Bidens by RP-HPLC. Huaxi Yaoxue Zazhi 2009, 24, 82-83.

134. Brandão, M.G.L.; Nery, C.G.C.; Mamão, M.A.S.; Krettli, A.U. Two methoxylated flavone glycosides from Bidens pilosa. Phytochemistry 1998, 48, 397-399.

135. Oliveira, F.Q.; Andrade-Neto, V.; Krettli, A.U.; Brandão, M.G.L. New evidences of antimalarial activity of Bidens pilosa roots extract correlated with polyacetylene and flavonoids. $J$. Ethnopharmacol. 2004, 93, 39-42.

136. Ogunbinu, A.O.; Flamini, G.; Cioni, P.L.; Adebayo, M.A.; Ogunwande, I.A. Constituents of Cajanus cajan (L.) Millsp., Moringa oleifera Lam., Heliotropium indicum L. and Bidens pilosa L. from Nigeria. Nat. Prod. Commun. 2009, 4, 573-578.

Sample Availability: Not available.

(C) 2011 by the authors; licensee MDPI, Basel, Switzerland. This article is an open access article distributed under the terms and conditions of the Creative Commons Attribution license (http://creativecommons.org/licenses/by/3.0/). 